\title{
ARTICLES
}

\section{Detection of anisotropies in the gravitational-wave stochastic background}

\author{
Bruce Allen* \\ Department of Physics, University of Wisconsin-Milwaukee, P.O. Box 413, Milwaukee, Wisconsin 53211 \\ Adrian C. Ottewill \\ Department of Mathematical Physics, University College Dublin, Belfield, Dublin 4, Ireland
}

(Received 26 July 1996; revised manuscript received 23 December 1996)

\begin{abstract}
By correlating the signals from a pair of gravitational-wave detectors, one can undertake sensitive searches for a stochastic background of gravitational radiation. If the stochastic background is anisotropic, then this correlated signal varies harmonically with Earth's rotation. We calculate how the harmonics of this varying signal are related to the multipole moments which characterize the anisotropy, and give a formula for the signal-to-noise ratio of a given harmonic. The specific case of the two LIGO (Laser Interferometric Gravitational Observatory) detectors, which will begin operation around the year 2000, is analyzed in detail. We consider two possible examples of anisotropy. If the gravitational-wave stochastic background contains a dipole intensity anisotropy whose origin (like that of the cosmic background radiation) is the motion of our local system, then that anisotropy will be observable by the advanced LIGO detector (with $90 \%$ confidence in one year of observation) if $\Omega_{\mathrm{GW}}>5.3 \times 10^{-8} h_{100}^{-2}$. We also study the signal produced by stochastic sources distributed in the same way as the luminous matter in the galactic disk, and in the same way as the galactic halo. The anisotropy due to sources distributed as the galactic disk or as the galactic halo will be observable by the advanced LIGO detector (with $90 \%$ confidence in one year of observation) if $\Omega_{\mathrm{GW}}>1.8 \times 10^{-10} h_{100}^{-2}$ or $\Omega_{\mathrm{GW}}>6.7 \times 10^{-8} h_{100}^{-2}$, respectively. [S0556-2821(97)02812-9]
\end{abstract}

PACS number(s): 04.80.Nn, 04.30.Db, 97.80.-d, 98.80.Es

\section{INTRODUCTION}

The design and construction of a number of new and more sensitive detectors of gravitational radiation is currently underway. These include the Laser Interferometric Gravitational Wave Observatory (LIGO) detector being built in the United States by a joint Caltech-MIT Collaboration [1], the VIRGO detector being built near Pisa by an Italian-French Collaboration [2], the GEO-600 detector being built in Hannover by an Anglo-German Collaboration [3], and the TAMA-300 detector being built near Tokyo [4]. There are also several resonant bar detectors currently in operation, and several more refined bar and interferometric detectors presently in the planning and proposal stages.

When two or more of these detectors are operating, it will become possible to correlate their signals, and in this way, to search for a stochastic background of gravitational radiation. The technique for such a search was originally described in work by Michelson [5], Cristensen [6], and Flanagan [7]. A review of these techniques may be found in [8]. Such radiation might be the result of processes that took place during the very early universe. It might also result from the incoherent superposition of many faint unresolvable present-day sources such as coalescing binary systems.

The stochastic gravitational-wave background might be

\footnotetext{
*Electronic address: ballen@dirac.phys.uwm.edu

†Electronic address: ottewill@ relativity.ucd.ie
}

isotropic on the sky, or it might be anisotropic. For example, if the background results from early-universe processes, then it might be isotropic to about the same degree as the $2.7 \mathrm{~K}$ electromagnetic background radiation. On the other hand, if the background is due to white-dwarf binaries in our own galaxy, then they might be distributed in a pancake or bar which mimics the shape of the observed luminous matter in our galaxy. In this paper, we show how the correlated signal from a pair of gravitational wave detectors is related to multipole moments which characterize the anisotropy. This should permit a signal to be analyzed to search for (or place upper limits on) the multipole moments which characterize the anisotropy. In this paper, we will assume that the reader is already familiar with the work previously cited (Refs. [5-8]) on stochastic background detection.

These paper is organized as follows. In Sec. II we show how a background of stochastic gravitational radiation may be decomposed in a plane-wave expansion, with the coefficients of the expansion treated as stochastic random variables. In Sec. III the properties of these random variables are related to the (frequency) spectrum and spatial distribution of the radiation, and a set of multipole moments are introduced which characterize the anisotropies of the stochastic background. These anisotropies may be searched for by studying the variations of the detector outputs as the Earth rotates relative to the fixed cosmic frame. In Sec. IV we show how the correlation between a pair of detectors fixed on the Earth varies with time as the Earth rotates, and detail how that correlation is related to the anisotropies of the stochastic 
gravitational background. The variation of the correlation with Earth's rotation may be decomposed into harmonics of Earth's period. In Sec. V we introduce a set of functions $\gamma_{\ell m}(f)$ which are generalizations of the well-known overlap reduction function $\gamma(f)$ of Refs. [5-8]. These functions characterize the effect of the $\ell, m$ anisotropy multipole on the $m$ th harmonic of the detector correlation. The principal result of this paper is to compute these functions for the LIGO pair of detectors. This is done by introducing two special frames of reference, fixed with respect to the Earth, in Sec. VI, and then performing a set of integrations in Sec. VII. The final integrations are performed and explicit formulas for the $\gamma_{/ m}(f)$ are obtained in Sec. VIII. In Sec. IX we analyze the signal-to-noise ratios associated with the $m$ th harmonic, and give a formula which may be used to determine if a given anisotropy is detectable or not. Following this, we consider two specific examples of anisotropy. In Sec. X we consider a dipole anisotropy in the stochastic background resulting from our local proper motion. In order to predict the harmonics which result, it is necessary to adopt conventions for the normalization of the optimal filters; these choices are detailed in Sec. XI. In Sec. XII we then consider the anisotropies in the stochastic gravitational wave background that would arise from sources distributed in the same way as our galaxy, and its halo. This is followed by a short conclusion. Throughout this paper, $c$ denotes the speed of light and $G$ denotes Newton's gravitational constant.

\section{THE STOCHASTIC BACKGROUND}

The gravitational wave background may be described in terms of a perturbation to the Minkowski metric of spacetime:

$$
d s^{2}=-c^{2} d t^{2}+d \vec{x}^{2}+h_{a b}(t, \vec{x}) d x^{a} d x^{b} .
$$

In transverse traceless gauge, this can be written in the form of a plane wave expansion as

$$
\begin{aligned}
h_{a b}(t, \vec{x})= & \sum_{A} \int_{-\infty}^{\infty} d f \int_{S^{2}} d \hat{\Omega} h_{A}(f, \hat{\Omega}) \\
& \times e^{2 \pi i f(t-\hat{\Omega} \cdot \vec{x} / c)} e_{a b}^{A}(\hat{\Omega}) .
\end{aligned}
$$

Here $h_{A}(f, \hat{\Omega})$ is an arbitrary complex function satisfying the relation $h_{A}(-f, \hat{\Omega})=h_{A}^{*}(f, \hat{\Omega})$. The polarization states are labeled by $A=+, \times$, and $\hat{\Omega}$ is a unit vector on the two sphere. The wave vector of the corresponding component of the perturbation is $\vec{k}=2 \pi f \hat{\Omega} / c$. The polarization tensors $e_{a b}^{A}$ appearing in these relations may be given explicitly. In standard angular coordinates $(\theta, \phi)$ on the two sphere one may write

$$
\begin{gathered}
\hat{\Omega}=\cos \phi \sin \theta \hat{x}+\sin \phi \sin \theta \hat{y}+\cos \theta \hat{z}, \\
\hat{m}=\sin \phi \hat{x}-\cos \phi \hat{y}, \\
\hat{n}=\cos \phi \cos \theta \hat{x}+\sin \phi \cos \theta \hat{y}-\sin \theta \hat{z}
\end{gathered}
$$

and then choose

$$
\begin{aligned}
& e_{a b}^{+}(\hat{\Omega})=m_{a} m_{b}-n_{a} n_{b}, \\
& e_{a b}^{\times}(\hat{\Omega})=m_{a} n_{b}+n_{a} m_{b} .
\end{aligned}
$$

To simplify matters later (but without any loss of generality) we assume that the $\hat{z}$ vector points along the direction of the Earth's rotation axis. One can verify by inspection that $\hat{m}$ and $\hat{n}$ are a pair of orthogonal unit-length vectors in the plane perpendicular to $\hat{\Omega}$. It is simple to show that any rotation of the vectors $\hat{m}$ and $\hat{n}$ within the plane that they define simply corresponds to a trivial redefinition of the complex wave amplitudes $h_{+}$and $h_{\times}$.

To describe a stochastic source, we treat the complex amplitude $h_{A}(f, \hat{\Omega})$ as a random variable with zero mean value. In this paper, we consider stochastic sources which are not isotropic. In principle, such a source has spectral properties which depends upon amplitude and frequency in an arbitrary way. For simplicity, in this paper we consider only stochastic sources whose directional dependence is frequency independent. The dependence of the stochastic background on frequency and direction may be stated in terms of the expectation value of the product of two random variables $h_{A}(f, \hat{\Omega})$ :

$$
\begin{aligned}
& \left\langle h_{A}^{*}(f, \hat{\Omega}) h_{A^{\prime}}\left(f^{\prime}, \hat{\Omega}^{\prime}\right)\right\rangle \\
& \quad=\delta_{A A^{\prime}} \delta\left(f-f^{\prime}\right) \delta^{2}\left(\hat{\Omega}, \hat{\Omega}^{\prime}\right) H(f) P(\hat{\Omega}) .
\end{aligned}
$$

Here $\delta^{2}\left(\hat{\Omega}, \hat{\Omega}^{\prime}\right)$ is a covariant two-dimensional deltafunction on the unit two-sphere. For a general stochastic source, the quantity $H(f) P(\hat{\Omega})$ which appears on the righthand side (RHS) would be an arbitrary function of frequency and direction. However, our assumption that the directional dependence is frequency independent implies that the RHS factors as shown. (Note that the expressions which we later derive for signal-to-noise ratios and expected signal strengths may be trivially extended to include the most general case.)

\section{SPECTRUM OF THE STOCHASTIC BACKGROUND}

The function $H(f)$ determines the spectrum of the gravitational radiation. The energy density in gravitational waves is given by

$$
\rho_{\mathrm{GW}}=\frac{c^{2}}{32 \pi G}\left\langle\dot{h}_{a b} \dot{h}^{a b}\right\rangle,
$$

where the overdot denotes a time derivative, and both tensors are evaluated at the same space-time point $(t, \vec{x})$. Substituting the plane wave expansion (2.2) into this formula and using Eq. (2.8) yields

$$
\begin{aligned}
& \left\langle\dot{h}_{a b}(t, \vec{x}) \dot{h}^{a b}(t, \vec{x})\right\rangle \\
& \quad=\sum_{A} \int_{-\infty}^{\infty} d f \int_{S^{2}} d \hat{\Omega} 4 \pi^{2} f^{2} H(f) P(\hat{\Omega}) e_{a b}^{A}(\hat{\Omega}) e_{A}^{a b}(\hat{\Omega}) .
\end{aligned}
$$

Since $\Sigma_{A} e_{a b}^{A} e_{A}^{a b}=4$ one has 


$$
\begin{aligned}
\left\langle\dot{h}_{a b} \dot{h}^{a b}\right\rangle & =16 \pi^{2} \int d \hat{\Omega} P(\hat{\Omega}) \int_{-\infty}^{\infty} d f f^{2} H(f) \\
& =32 \pi^{2} \int d \hat{\Omega} P(\hat{\Omega}) \int_{0}^{\infty} d f f^{2} H(f) .
\end{aligned}
$$

In describing gravitational wave stochastic backgrounds, it is conventional to compare the energy density to the critical energy density $\rho_{\text {critical }}$ required (today) to close the universe. This critical energy density is determined by the rate at which the universe is expanding today. Let us denote the Hubble expansion rate today by

$$
\begin{aligned}
H_{0} & =100 h_{100} \frac{\mathrm{km} \mathrm{sec}^{-1}}{\mathrm{Mpc}}=3.2 \times 10^{-18} h_{100} \mathrm{sec}^{-1} \\
& =1.1 \times 10^{-28} \mathrm{ch}_{100} \mathrm{~cm}^{-1} .
\end{aligned}
$$

The value of $H_{0}$ is determined by the dimensionless factor of $h_{100}$ which probably lies within the range $1 / 2<h_{100}<1$. The critical energy density required to just close the universe is

$$
\rho_{\text {critical }}=\frac{3 c^{2} H_{0}^{2}}{8 \pi G} \approx 1.6 \times 10^{-8} h_{100}^{2} \mathrm{ergs} / \mathrm{cm}^{3} .
$$

The spectrum of an isotropic stochastic gravitational wave background is defined by a dimensionless function of frequency $f$ :

$$
\Omega_{\mathrm{GW}}(f) \equiv \frac{1}{\rho_{\text {critical }}} \frac{d \rho_{\mathrm{GW}}}{d \ln f} .
$$

Here $d \rho_{\mathrm{GW}}$ is the energy density in gravitational waves contained within the frequency interval $(f, f+d f)$. Using the definition $\Omega_{\mathrm{GW}}$ one obtains the relationship between the spectrum $\Omega_{\mathrm{GW}}$ and $H(f)$. For $f \geqslant 0$ one has

$$
\begin{aligned}
\Omega_{\mathrm{GW}}(f) & =\frac{f}{\rho_{\text {critical }}} \frac{d \rho_{\mathrm{GW}}}{d f} \\
& =f \frac{8 \pi G}{3 c^{2} H_{0}^{2}} \frac{c^{2}}{32 \pi G} 32 \pi^{2} f^{2} H(f) \int d \hat{\Omega} P(\hat{\Omega}) \\
& =\frac{8 \pi^{2}}{3 H_{0}^{2}} f^{3} H(f) \int d \hat{\Omega} P(\hat{\Omega}) .
\end{aligned}
$$

This formula shows the precise interpretation of $P(\hat{\Omega})$. The stochastic background energy density is made of contributions arriving from all directions $\hat{\Omega}$ on the sky. The actual value of $\Omega_{\mathrm{GW}}(f)$ is determined by the average value of $P(\hat{\Omega})$; the direction dependence of this function is the same as the direction dependence of the arriving radiation intensity.

For this reason, we define the multipole moments $p_{\ell m}$ of the stochastic background radiation by the expansion of $P(\hat{\Omega})$ in terms of spherical harmonic functions:

$$
P(\hat{\Omega}) \equiv \sum_{\ell m} p_{\ell m} Y_{\ell m}(\hat{\Omega})
$$

where the sum is defined by

$$
\sum_{\ell m} \equiv \sum_{\ell=0}^{\infty} \sum_{m=-\ell}^{\ell}=\sum_{m=-\infty}^{\infty} \sum_{\ell=|m|}^{\infty} .
$$

In addition, without loss of generality we adopt the convention that the monopole moment is normalized by the condition

$$
p_{00} \equiv \sqrt{4 \pi} \Rightarrow \int d \hat{\Omega} P(\hat{\Omega}) \equiv 4 \pi,
$$

where we assume that the spherical harmonic functions are normalized in the conventional way, so that the integrals of their squares over the unit sphere gives unity. Hence the spectrum of radiation is determined entirely by $H(f)$ since for $f \geqslant 0$ one has

$$
\Omega_{\mathrm{GW}}(f)=\frac{32 \pi^{3}}{3 H_{0}^{2}} f^{3} H(f) .
$$

The directionality of the arriving radiation is determined entirely by the function $P(\hat{\Omega})$. Our fundamental assumption here is that the pattern of the intensity of the stochastic background is fixed in a frame of reference at rest with respect to the cosmological fluid. In other words, formula (3.8) for $P(\hat{\Omega})$ is expressed in a set of coordinates $x, y, z$ which are fixed with respect to the distant stars. In those coordinates, the multipole moments $p_{\ell m}$ are constants, independent of time. The problem we address in this paper is this: how do we determine, from the data stream of a pair of interferometric detectors which are rotating with the Earth, the values of (or bounds on) the multipole moments $p_{\ell m}$ ?

\section{DETECTION STRATEGY}

To determine the multipole moments $p_{\ell m}$ the basic idea is to correlate the outputs of two gravitational wave detectors, and to look for variations of this correlated signal that are harmonics of Earth's rotational frequency. For this purpose, we need to consider the relationship between two different time (or frequency) scales that occur.

The first time scale is that defined by the light travel time $\Delta T$ between the two sites. For the remainder of this section, we will assume that the two sites are the Hanford and Livingston LIGO detectors, so that $\Delta T=10.00 \mathrm{msec}$. The second time scale is the period of Earth's rotation about its axis relative to the cosmic frame, $T_{\mathrm{e}}=8.6 \times 10^{4} \mathrm{sec}=1$ sidereal day. Because of the enormous disparity between these two time scales, we can define a third time scale, which we will refer to as the averaging time scale $\tau$. We choose $\tau$ in the range

$$
\Delta T \ll \tau \ll T_{\mathrm{e}},
$$

for example, $\tau=30 \mathrm{sec}$. It is then possible to examine correlations between the two detectors as a function of time, averaged over periods of length $\tau$. Because $\tau$ is much shorter than $T_{\mathrm{e}}$, the correlation between the two detectors will vary as the Earth rotates relative to the fixed cosmological frame, because of the anisotropy in $P(\hat{\Omega})$. On the other hand, because $\tau$ is much longer than the light travel time between the 
two detectors, and because the detectors are sensitive to frequencies $f \approx 1 / \Delta T$, there is a significant correlated signal on time scales shorter than $\tau$.

Denote the output of the first detector by

$$
s_{1}(t)=h_{1}(t)+n_{1}(t),
$$

where $h_{1}$ is the strain due to the stochastic background and $n_{1}$ is the intrinsic noise of the first detector. In similar fashion, the output of the second detector is

$$
s_{2}(t)=h_{2}(t)+n_{2}(t) .
$$

Let us use the subscript $i=1,2$ to label the detectors, so, for example, $i=1$ denotes the Hanford, WA LIGO detector and $i=2$ denotes the Livingston, LA LIGO detector. The response $h_{i}$ of detector $i$ to the gravitational radiation is given by

$$
h_{i}(t)=d_{i}^{a b}(t) h_{a b}\left[t, \vec{x}_{i}(t)\right],
$$

where the position of detector $i$ 's corner station is denoted by $\vec{x}_{i}(t)$. In this expression, the symmetric traceless tensors $d_{i}^{a b}(t)$ are given by

$$
d_{i}^{a b}(t)=\frac{1}{2}\left[\hat{X}_{i}^{a}(t) \hat{X}_{i}^{b}(t)-\hat{Y}_{i}^{a}(t) \hat{Y}_{i}^{b}(t)\right],
$$

where the directions of detector $i$ 's arms are defined by the unit spatial vectors $\hat{X}_{i}^{a}(t)$ and $\hat{Y}_{i}^{a}(t)$. Note that $b o t h d_{i}^{a b}(t)$ and $\vec{x}_{i}(t)$ are functions of time, because the Earth rotates with respect to the cosmological rest frame.

Define quantities which are the Fourier transforms of the signals, evaluated over an interval of one averaging time $\tau$ centered at time $t$ :

$$
\widetilde{s_{i}}(f, t)=\int_{t-\tau / 2}^{t+\tau / 2} d t^{\prime} e^{-2 \pi i f t^{\prime}} s_{i}\left(t^{\prime}\right) \text { for } i=1,2 .
$$

These Fourier transforms are easily evaluated. Substituting the plane wave expansion (2.2) into the formula for the strain (4.4) and taking the Fourier transform (4.6) we obtain

$$
\begin{aligned}
\widetilde{s}_{i}(f, t)= & \sum_{A} \int d \hat{\Omega} \int_{-\infty}^{\infty} d f^{\prime} e^{2 \pi i\left(f^{\prime}-f\right) t} \delta_{\tau}\left(f-f^{\prime}\right) \\
& \times h_{A}\left(f^{\prime}, \hat{\Omega}\right) d_{i}^{a b}(t) e_{a b}^{A}(\hat{\Omega}) e^{-2 \pi i f^{\prime} \hat{\Omega} \cdot \vec{x}_{i}(t) / c} \\
& + \text { noise term. }
\end{aligned}
$$

In this expression, we have made use of the fact that the averaging time $\tau$ is much less than the rotation period of the Earth $\Delta T_{\mathrm{e}}$, so that the vectors $X_{i}^{a}(t), Y_{i}^{a}(t)$, and $\vec{x}_{i}(t)$ may be treated as constants and taken outside of the time integration in the Fourier transform (4.6). We have also defined the "finite time" approximation to the Dirac delta function

$$
\delta_{\tau}(f) \equiv \int_{-\tau / 2}^{\tau / 2} d t^{\prime} e^{-2 \pi i f t^{\prime}}=\frac{\sin (\pi f \tau)}{\pi f},
$$

which reduces to the Dirac delta function $\delta(f)$ in the limit $\tau \rightarrow \infty$, but has the property that $\delta_{\tau}(0)=\tau$. The final term on the right-hand side of Eq. (4.7) is linearly proportional to the noise in detector $i$.

We now define the "signal"

$$
S(t)=\int_{-\infty}^{\infty} d f \widetilde{s}_{1}^{*}(f, t) \widetilde{s}_{2}(f, t) \widetilde{Q}(f)
$$

where $\widetilde{Q}(f)$ is an optimal filter function, to be determined. Let us now determine the expectation value of $S(t)$ and show how it incorporates information about the multipole moments of the stochastic background. To find the expected value $S(t)$ we begin by assuming that the noise in each detector has zero mean value, and is uncorrelated with noise and gravitational strain in the other detector. Under these assumptions, we find

$$
\begin{aligned}
\langle S(t)\rangle= & \int_{-\infty}^{\infty} d f \widetilde{Q}(f) \sum_{A} \sum_{A^{\prime}} \int_{-\infty}^{\infty} d f^{\prime} \int_{-\infty}^{\infty} d f^{\prime \prime} \int d \hat{\Omega} \\
& \times \int d \hat{\Omega}^{\prime} e^{-2 \pi i\left(f^{\prime}-f\right) t} e^{2 \pi i\left(f^{\prime \prime}-f\right) t} \delta_{\tau}\left(f-f^{\prime}\right) \delta_{\tau}\left(f-f^{\prime \prime}\right) \\
& \times d_{1}^{a b}(t) d_{2}^{c d}(t) e_{a b}^{A}(\hat{\Omega}) e_{c d}^{A^{\prime}}\left(\hat{\Omega}^{\prime}\right) \exp \left\{2 \pi i \left[f^{\prime} \hat{\Omega} \cdot \vec{x}_{1}(t)\right.\right. \\
& \left.\left.-f^{\prime \prime} \hat{\Omega}^{\prime} \cdot \vec{x}_{2}(t)\right] / c\right\}\left\langle h_{A}^{*}\left(f^{\prime}, \hat{\Omega}\right) h_{A^{\prime}}\left(f^{\prime \prime}, \hat{\Omega}^{\prime}\right)\right\rangle .
\end{aligned}
$$

We now substitute in the expectation value for the product of the amplitudes (2.8). The integration over $f^{\prime \prime}$ is now trivial. In the resulting expression, because $1 / \tau$ is much smaller than the "bandwidth" $1 / \Delta T$ of the signals, one of the finite-width delta functions $\delta_{\tau}$ may be replaced by a Dirac $\delta$ function. The integration over $f^{\prime}$ is then trivial. The other finite-width $\delta$ function is then evaluated at zero argument, giving rise to a factor of $\tau$. One thus obtains

$$
\begin{aligned}
\langle S(t)\rangle= & \tau d_{1}^{a b}(t) d_{2}^{c d}(t) \int_{-\infty}^{\infty} d f \widetilde{Q}(f) H(f) \\
& \times \int d \hat{\Omega} P(\hat{\Omega}) \mathrm{e}^{2 \pi i f \hat{\Omega} \cdot \Delta \vec{x}(t) / c} \sum_{A} e_{a b}^{A}(\hat{\Omega}) e_{c d}^{A}(\hat{\Omega}),
\end{aligned}
$$

where $\Delta \vec{x}(t)=\vec{x}_{1}(t)-\vec{x}_{2}(t)$ is the time-dependent separation vector between the two interferometer sites.

Not surprisingly, this previous expression can be easily simplified for the isotropic case $P(\hat{\Omega})=1$. In this instance, the sum over polarizations and integral over directions can be performed explicitly, yielding ( $8 \pi / 5$ times) a timeindependent function of frequency known as the overlap reduction function $\gamma(f)$. This overlap reduction function is given by

$$
\begin{aligned}
\gamma(f) \equiv & \frac{5}{8 \pi} d_{1}^{a b} d_{2}^{c d} \int_{S^{2}} d \hat{\Omega} e^{2 \pi i f \hat{\Omega} \cdot \Delta \vec{x} / c} \\
& \times\left[e_{a b}^{+}(\hat{\Omega}) e_{c d}^{+}(\hat{\Omega})+e_{a b}^{\times}(\hat{\Omega}) e_{c d}^{\times}(\hat{\Omega})\right] .
\end{aligned}
$$

Notice that in Eq. (4.11) the dependence of the positions and orientations of the detectors upon time $t$ is not shown; this is because $\gamma(f)$ depends only upon the relative positions and orientations, which is time (or Earth-position) independent. Thus, in the case of an isotropic stochastic background, one finds 


$$
P(\hat{\Omega})=1 \Rightarrow\langle S(t)\rangle=\frac{8 \pi}{5} \tau \int_{-\infty}^{\infty} d f \widetilde{Q}(f) H(f) \gamma(f) .
$$

This is Eq. (30) of Ref. [8]. In the present paper, we are most interested in the anisotropic case where $P(\hat{\Omega})$ varies with direction. In this case, the time variation of the tensors $d_{i}^{a b}(t)$ and $\Delta \vec{x}(t)$ will provide a time-dependent variation of the signal $S(t)$.

\section{ROTATION HARMONICS}

Because the rotation of the Earth is periodic with period $T_{\mathrm{e}}$ and angular frequency $\omega_{\mathrm{e}}=2 \pi / T_{\mathrm{e}}$ the expected signal (4.10) varies with the same period. It can therefore be represented by the Fourier series

$$
\langle S(t)\rangle=\sum_{m=-\infty}^{\infty}\left\langle S_{m}\right\rangle e^{i m \omega_{\mathrm{e}} t}
$$

Because the signal is real, the amplitudes of the different harmonics satisfy $S_{m}=S_{-m}^{*}$. The amplitudes are quantities which would be determined by Fourier transforming the actual data:

$$
S_{m}=\frac{1}{T} \int_{0}^{T} d t e^{-i m \omega_{\mathrm{e}} t} S(t)
$$

Here $T$ represents the total observation time, which later enters into Eq. (9.4) and which is assumed to be is a multiple of Earth's rotation period $T_{\mathrm{e}}$. The harmonic amplitudes $S_{m}$ are the (at least in principle) observable quantities on which any data analysis must be based; it is their expected values $\left\langle S_{m}\right\rangle$ which arise in the formula for the expected signal. Note that in an actual observation or measurement, instrumental noise in the gravitational-wave detectors would prevent $S_{m}$ from taking on its expected value. In Sec. IX we analyze the typical deviations of $S_{m}$ from $\left\langle S_{m}\right\rangle$ and in this way determine how accurately $S_{m}$ may in fact be measured.

Because we have assumed that the $z$ axis of our (cosmic) coordinate system points along the direction of Earth's axis, the $m$ th rotation harmonic can only result from anisotropies whose phase varies with angle $\phi$ as $\exp (\operatorname{im} \phi)$. These are the anisotropies associated with the $Y_{\ell m}$. Hence

$$
\left\langle S_{m}\right\rangle=\frac{8 \pi}{5} \tau \int_{-\infty}^{\infty} d f \widetilde{Q}(f) H(f) \sum_{\ell=|m|}^{\infty} p_{\ell m} \gamma_{\ell m}(f) .
$$

The functions $\gamma_{\ell m}(f)$ are generalizations of the overlap reduction function $\gamma(f)$, which express the (frequencydependent) contribution of the $\ell$ th multipole moment to the $m$ th harmonic of the signal, with respect to Earth's rotation. These are given by

$$
\begin{aligned}
\gamma_{\ell m}(f)= & \frac{5}{8 \pi} \frac{1}{2 \pi} \int_{0}^{2 \pi} d \alpha e^{-i m \alpha} d_{1}^{a b}(\alpha) d_{2}^{c d}(\alpha) \\
& \times \int d \hat{\Omega} \quad Y_{\ell m}(\hat{\Omega}) e^{2 \pi i f \hat{\Omega} \cdot \Delta \vec{x}(\alpha) / c} \\
& \times \sum_{A} e_{a b}^{A}(\hat{\Omega}) e_{c d}^{A}(\hat{\Omega}) .
\end{aligned}
$$

In this expression, the angle of rotation of the earth about its axis (measured from some arbitrary fiducial point) is denoted by $\alpha \in[0,2 \pi)$ so $\alpha=\omega_{e} t+$ const $(\bmod 2 \pi)$. The "timedependent" quantities $d_{i}^{a b}$ and $\Delta \vec{x}$ may equivalently be expressed as functions of $\alpha$.

The problem at hand is now a mathematical one-to calculate the functions $\gamma_{\ell m}(f)$ which are generalizations of the overlap reduction function $\gamma(f)$. For the monopole moment $(\ell=m=0)$ it is easy to see that the integrand above is independent of Earth position $\alpha$ because the overlap reduction function (4.11) only depends upon the relative orientations of the detectors, which is $\alpha$ independent, giving

$$
\gamma_{00}(f)=(4 \pi)^{-1 / 2} \gamma(f) .
$$

In the next parts of this paper, we will show how to evaluate the other $\gamma_{\ell m}$.

Our first task is to evaluate the integrals that appear in Eq. (5.4). The product $d_{1}^{a b}(\alpha) d_{2}^{c d}(\alpha)$ is a quartic polynomial in $\sin \alpha$ and $\cos \alpha$. One approach would be to attempt to perform the integral over $\hat{\Omega}$, to obtain the resulting function of $\alpha$, and then to evaluate the integral over $\alpha$. However, this approach is rather cumbersome.

A more promising method is to consider the projector onto the plane perpendicular to $\hat{\Omega}$, which may be calculated in terms of the vectors defined by Eqs. (2.3) and (2.4):

$$
Q_{a b}=\delta_{a b}-\hat{\Omega}_{a} \hat{\Omega}_{b}=\hat{m}_{a} \hat{m}_{b}+\hat{n}_{a} \hat{n}_{b} .
$$

A couple minutes of algebra starting with Eqs. (2.6) and (2.7) quickly establishes the identity

$$
\sum_{A} e_{a b}^{A}(\hat{\Omega}) e_{c d}^{A}(\hat{\Omega})=Q_{a c} Q_{b d}+Q_{a d} Q_{b c}-Q_{a b} Q_{c d} .
$$

We then define the set of integrals

$$
C_{a b c d}(\alpha)=\int d \hat{\Omega} \quad Y_{\ell m}(\hat{\Omega}) e^{2 \pi i f \hat{\Omega} \cdot \Delta \vec{x}(\alpha) / c} \hat{\Omega}_{a} \hat{\Omega}_{b} \hat{\Omega}_{c} \hat{\Omega}_{d} .
$$

The desired integrals can then be expressed in terms of this quantity. For convenience, we introduce a symbol to handle the contractions that occur. This is a constant tensor defined by

$$
\Theta_{a b c d}^{p q r s}=2 \delta_{a c} \delta_{b d} \delta^{p q} \delta^{r s}-4 \delta_{a c} \delta^{p q} \delta_{b}^{r} \delta_{d}^{s}+\delta_{a}^{p} \delta_{b}^{q} \delta_{c}^{r} \delta_{d}^{s}
$$

Making use of the fact that each of the $d_{i}^{a b}$ is symmetric in its tensor indices, and traceless, we may then write

$$
\begin{aligned}
\gamma_{\ell m}(f)= & \frac{5}{8 \pi} \frac{1}{2 \pi} \int_{0}^{2 \pi} d \alpha e^{-i m \alpha} d_{1}^{a b}(\alpha) \\
& \times d_{2}^{c d}(\alpha) \Theta_{a b c d}^{p q r s} C_{p q r s}(\alpha) .
\end{aligned}
$$

From this definition it is easy to show that $\gamma_{\ell,-m}=(-1)^{\ell+m} \gamma_{\ell, m}^{*}$. This follows from the parity transformation property of the spherical harmonics 
$Y_{\ell m}(-\hat{\Omega})=(-1)^{\ell} Y_{\ell m}(\hat{\Omega})$. In order to now evaluate $C_{a b c d}$ it is convenient to introduce some additional coordinate systems.

\section{COORDINATE FRAMES}

The vectors being used in this calculation are threedimensional spatial vectors in flat Cartesian $R^{3}$. Up to this point, we have been using a coordinate system which is fixed with respect to the cosmological fluid, and in which the spatial pattern of the perturbations of the stochastic background is assumed to be time independent. This frame of reference is the "unprimed" frame; vectors expressed with respect to these cosmic coordinates have unprimed indices. We have also assumed (without any loss of generality) that the $z$ axis of this cosmic frame points along the direction of Earth's rotation axis.

At this point, for calculational purposes, it is convenient to consider two additional coordinate systems. Thus, a given vector $V$ may be expressed in terms of its components in three different frames:

\section{Cosmic frame: $V^{a}$, Earth frame: $V^{\bar{a}}$, \\ Computational frame: $V^{a^{\prime}}$.}

The "Earth frame" is a coordinate system fixed to the Earth, in which the third ( $z$ coordinate) points along the axis of Earth's rotation, in the direction of the north pole. Components of vectors in this frame are denoted with "barred" indices. The second of these new coordinate systems will be referred to as the "calculational" coordinate system. In this frame, the components of vectors are "primed." This frame is fixed with respect to the Earth, and has its third ( $z$ coordinate) pointing along the line between the two gravitationalwave detectors.

The relationship between components of vectors in these three coordinate frames may be written as matrix equations. Each of the matrices which appears is a special case of a rotation matrix which may be parametrized by Euler angles. Throughout this paper, we use the Euler angle conventions given by Eqs. (4.83)-(4.86) of Afkin [9] which are also the conventions used in Eqs. (4.5) and (4.43) of Rose [10]. It is convenient to define a pair of rotations about the $z$ and $y$ axes, respectively, by

$$
\begin{aligned}
& \mathbf{R}_{z}(\alpha) \equiv\left(\begin{array}{ccc}
\cos \alpha & \sin \alpha & 0 \\
-\sin \alpha & \cos \alpha & 0 \\
0 & 0 & 1
\end{array}\right), \\
& \mathbf{R}_{y}(\beta) \equiv\left(\begin{array}{ccc}
\cos \beta & 0 & -\sin \beta \\
0 & 1 & 0 \\
\sin \beta & 0 & \cos \beta
\end{array}\right) .
\end{aligned}
$$

The most general possible rotation may be parametrized by Euler angles and is defined by the matrix $\mathbf{R}(\alpha, \beta, \gamma)=\mathbf{R}_{z}(\gamma) \mathbf{R}_{y}(\beta) \mathbf{R}_{z}(\alpha)$. Note that the boldface symbols here denote $3 \times 3$ square matrices.
The matrix which relates components of vectors in the cosmic and Earth frames is simply rotation through angle $\alpha$ about the $z$ axis:

$$
X^{\bar{a}}=R_{a}^{\bar{a}} X^{a}, \quad R_{a}^{\bar{a}}=\mathbf{R}(\alpha, 0,0)=\mathbf{R}_{z}(\alpha),
$$

where the first index on $R$ labels rows and the second index labels columns, so that the operation appearing in the previous equation is ordinary multiplication of a column vector on the right by a square matrix on the left. Note that the angle $\alpha=\omega_{\mathrm{e}} t$ varies with time.

Without loss of generality, assume that the freedom to choose the $\bar{x}$ and $\bar{y}$ axis in the Earth frame has been used to ensure that in this frame the separation vector $\Delta x^{\bar{a}}$ between the two detector sites has no $\bar{y}$ component. Using the two LIGO sites as an example, the Earth-frame $\bar{x}$ axis would point out from the center of the Earth at an angle $38.6881^{\circ}$ East of the $0^{\circ}$ line of longitude (Greenwich, England). In this frame, the coordinates of the two detector sites and the detector arms directions are

$$
\begin{gathered}
\text { Hanford, Washington: } x_{1}^{\bar{a}}=\left(\begin{array}{c}
707.41 \\
-4329.11 \\
4614.74
\end{array}\right)^{\bar{a}} \mathrm{~km}, \\
\hat{X}_{1}^{\bar{a}}=\left(\begin{array}{c}
-0.684779 \\
0.476172 \\
0.55167
\end{array}\right)^{\bar{a}}, \\
\hat{Y}_{1}^{\bar{a}}=\left(\begin{array}{c}
-0.720231 \\
-0.557622 \\
-0.412703
\end{array}\right)^{\bar{a}}, \\
\text { Livingston, Louisiana: } x_{2}^{\bar{a}}=\left(\begin{array}{c}
3371.80 \\
-4329.11 \\
3240.36
\end{array}\right)^{\bar{a}} \mathrm{~km}, \\
\hat{X}_{2}^{\bar{a}}=\left(\begin{array}{c}
-0.65377 \\
-0.708366 \\
-0.266085
\end{array}\right)^{\bar{a}} \\
\hat{Y}_{2}^{\bar{a}}=\left(\begin{array}{c}
0.540953 \\
-0.191642 \\
-0.81893
\end{array}\right)^{\bar{a}} .
\end{gathered}
$$

It is obvious that, as claimed, the separation vector between the two sites $x_{1}^{\bar{a}}-x_{2}^{\bar{a}}$ has vanishing $\bar{y}$ component. The matrix which relates the components of the vectors in the computational and Earth frames is a rotation about the $\bar{y}$ axis:

$$
X^{\bar{a}}=R_{a}^{\bar{a}}, X^{a^{\prime}}, \quad R_{a}^{\bar{a}}=\mathbf{R}(0,-\beta, 0)=\mathbf{R}_{y}(-\beta),
$$

where $\beta$ is a time-independent (or $\alpha$-independent) angle, determined by the relative orientation of the line between the 
two detector sites and Earth's axis. For the two LIGO detectors, the angle relating the Earth frame and the computational frame is

$$
\beta=-62.71383^{\circ}
$$

Within the computational frame, the separation vector between the two sites is

$$
x_{1}^{a^{\prime}}-x_{2}^{a^{\prime}}=2997.98 \mathrm{~km}\left(\begin{array}{l}
0 \\
0 \\
1
\end{array}\right)
$$

and the unit-length vectors defining the arm directions are

$$
\begin{gathered}
\hat{X}_{1}^{a^{\prime}}=\left(\begin{array}{c}
0.176358 \\
0.476171 \\
0.861486
\end{array}\right), \quad \hat{Y}_{1}^{a^{\prime}}=\left(\begin{array}{c}
-0.69696 \\
-0.557623 \\
0.450893
\end{array}\right)^{a^{\prime}}, \\
\hat{X}_{2}^{a^{\prime}}=\left(\begin{array}{c}
-0.536188 \\
-0.708366 \\
0.459042
\end{array}\right)^{a^{\prime}}, \quad \hat{Y}_{2}^{a^{\prime}}=\left(\begin{array}{r}
-0.479814 \\
-0.191642 \\
-0.856185
\end{array}\right)^{a^{\prime}} .
\end{gathered}
$$

These quantities will become useful later.

\section{COMPUTATION OF $C$}

Our goal now is to calculate $C_{a b c d}$ as defined in Eq. (5.8). To do this, we will express the spherical harmonic functions $Y_{\ell m}(\Omega)=Y_{\ell m}(\theta, \phi)$ in terms of the "primed" coordinates in the computational frame. Combining the transformations (6.2) and (6.3) we obtain the relationship between vectors in the cosmic and computational frames:

$$
V^{a^{\prime}}=\mathbf{R}_{y}(\beta) \mathbf{R}_{z}(\alpha) V^{a}=\mathbf{R}(\alpha, \beta, 0) V^{a} .
$$

This transformation through Euler angles $\alpha, \beta, 0$ induces a simple change in the spherical harmonics. For a given value of $\ell$ the spherical harmonic functions in one frame are simply a sum of all the spherical harmonics with the same value of $\ell$ in the other frame. The relation between these two sets of functions is given by the rotation matrices $D_{m n}^{l}$, which are closely related to Clebsch-Gordan coefficients

$$
Y_{\ell m}\left(\theta^{\prime}, \phi^{\prime}\right)=\sum_{k=-\ell}^{\ell} D_{k m}^{\ell}(\alpha, \beta, 0) Y_{\ell k}(\theta, \phi) .
$$

(See equation (4.260) of Arfkin [9].) The inverse transformation is obtained by reversing the lower two indices on the rotation matrix and complex conjugating:

$$
Y_{\ell m}(\theta, \phi)=\sum_{k=-\ell}^{\ell}\left[D_{m k}^{\ell}(\alpha, \beta, 0)\right]^{*} Y_{\ell k}\left(\theta^{\prime}, \phi^{\prime}\right) \text {. }
$$

The rotation matrices are conveniently expressed by Eq. (4.12) of Rose [10]:

$$
D_{m k}^{\ell}(\alpha, \beta, \gamma)=e^{-i m \alpha} d_{m k}^{\ell}(\beta) e^{-i k \gamma}
$$

Explicit formulas for the $d_{m k}^{\ell}$ may be given either in the form of a sum or in "summed" form. The latter expression, given by Eq. (4.14) of Rose [10] is the most useful one for us. For $m \geqslant k$,

$$
\begin{aligned}
d_{m k}^{\ell}= & {\left[\frac{(\ell-k) !(\ell+m) !}{(\ell+k) !(\ell-m) !}\right]^{1 / 2} } \\
& \times \frac{(\cos \beta / 2)^{2 \ell+k-m}(-\sin \beta / 2)^{m-k}}{(m-k) !} \\
& \times{ }_{2} F_{1}\left(m-\ell,-k-\ell ; m-k+1 ;-\tan ^{2} \frac{\beta}{2}\right) .
\end{aligned}
$$

Notice that because the $m \leqslant \ell$ the first argument of the Gauss hypergeometric function ${ }_{2} F_{1}$ is a nonpositive integer the hypergeometric series ${ }_{2} F_{1}$ terminates after a finite number of terms. In fact it is possible to rewrite Eq. (7.5) in terms of Jacobi polynomials $P_{n}^{(\alpha, \beta)}$ [11], for $m \geqslant k$

$$
\begin{aligned}
d_{m k}^{\ell}= & (-1)^{l-m}\left[\frac{(\ell-m) !(\ell+m) !}{(\ell-k) !(\ell+k) !}\right]^{1 / 2}\left(\cos \frac{\beta}{2}\right)^{m+k} \\
& \times\left(-\sin \frac{\beta}{2}\right)^{m-k} P_{\ell-m}^{(m+k, m-k)}(-\cos \beta) .
\end{aligned}
$$

In the event that $m<k$ the $d_{m k}^{\ell}$ may be obtained from the unitarity property, Eq. (4.15) of Rose [10],

$$
d_{m k}^{\ell}(\beta)=d_{k m}^{\ell}(-\beta)=(-1)^{m-k} d_{k m}^{\ell}(\beta) .
$$

Note also that the $d_{m k}^{\ell}$ are real, so that we can drop the complex conjugation that would otherwise have appeared.

The integral over the two-sphere which appears in Eq. (5.8) can also be expressed as an integral over all directions in the computational (primed) frame. In other words, $\int d \hat{\Omega}=\int d \hat{\Omega}^{\prime}$. So our integral may be expressed as

$$
\begin{aligned}
C_{a b c d}(\alpha)= & \sum_{k=-\ell}^{\ell} d_{m k}^{\ell}(\beta) \times e^{i m \alpha} N_{\ell}^{k} \\
& \times \int_{0}^{\pi} \sin \theta^{\prime} d \theta^{\prime} e^{2 \pi i f \Delta T \cos \theta^{\prime}} P^{k}\left(\cos \theta^{\prime}\right) \\
& \times \int_{0}^{2 \pi} d \phi^{\prime} e^{i k \phi^{\prime}} \hat{\Omega}_{a} \hat{\Omega}_{b} \hat{\Omega}_{c} \hat{\Omega}_{d},
\end{aligned}
$$

where we have expressed the spherical harmonic functions in terms of associated Legendre functions $P_{\ell}^{k}$, and $\Delta T=\left|x_{1}-x_{2}\right| / c$ denotes the light travel time between the two detector sites (10.00 msec for the two LIGO detectors). The normalization constants $N_{\ell}^{k}$ which relate the spherical harmonics and the Legendre polynomials are

$$
N_{\ell}^{k}=\sqrt{\frac{2 l+1}{4 \pi} \frac{(l-k) !}{(l+k) !}} .
$$

We will eventually be contracting the four indices of $C$ with each other and with the indices of other tensors. Of course such contractions yield the same result in any coordinate frame, and it is easier to calculate $C$ in the 
calculational (primed) frame. Hence we may write $C_{a b c d}(\alpha)=R_{a}^{a \prime} R_{b}^{b \prime} R_{c}^{c \prime} R_{d}^{d \prime} C_{a^{\prime} b^{\prime} c^{\prime} d^{\prime}}, \quad$ where $\quad R_{a}^{a \prime}$ $=\mathbf{R}_{z}(-\alpha) \mathbf{R}_{y}(-\beta)$, and

$$
\begin{aligned}
C_{a^{\prime} b^{\prime} c^{\prime} d^{\prime}}= & e^{i m \alpha} \sum_{k=-\ell}^{\ell} d_{m k}^{\ell}(\beta) N_{\ell}^{k} \int_{0}^{\pi} \sin \theta^{\prime} d \theta^{\prime} \\
& \times e^{2 \pi i f \Delta T \cos \theta^{\prime}} P^{k}\left(\cos \theta^{\prime}\right) \\
& \times \int_{0}^{2 \pi} d \phi^{\prime} e^{i k \phi^{\prime}} \hat{\Omega}_{a^{\prime}} \hat{\Omega}_{b^{\prime}} \hat{\Omega}_{c^{\prime}} \hat{\Omega}_{d^{\prime}}
\end{aligned}
$$

The vector $\hat{\Omega}^{a^{\prime}}$ is

$$
\hat{\Omega}^{a^{\prime}}=\left(\begin{array}{c}
\cos \phi^{\prime} \sin \theta^{\prime} \\
\sin \phi^{\prime} \sin \theta^{\prime} \\
\cos \theta^{\prime}
\end{array}\right)^{a^{\prime}}
$$

It is clear that the integral over $\phi^{\prime}$ in Eq. (7.10) vanishes unless $k=-4,-3, \ldots, 3,4$. Thus even for large $\ell$ the range of summation over $k$ only includes these values. There is a sense in which this reflects the fact that our signal is a product of the outputs of a pair of detectors, each of which has a quadrupole antenna pattern. It is also noteworthy that the remaining integral, over the variable $\theta^{\prime}$, can also be done explicitly for any distinct values of $\ell$ and $k$.

\section{THE REMAINING INTEGRATIONS}

We are now in a position to evaluate the remaining integrals. We begin with the integral over $\alpha$. We can rewrite $\gamma_{\ell m}$ from Eq. (5.10) as

$$
\begin{aligned}
\gamma_{\ell m}(f)= & \frac{5}{8 \pi} \frac{1}{2 \pi} \int_{0}^{2 \pi} d \alpha e^{-i m \alpha} e^{i m \alpha} d_{1}^{a^{\prime} b^{\prime}}(\beta) d_{2}^{c^{\prime} d^{\prime}}(\beta) \\
& \times \Theta_{a^{\prime} b^{\prime} c^{\prime} d^{\prime}}^{p^{\prime} q^{\prime} r^{\prime} s^{\prime}} \sum_{k=-\ell}^{\ell} d_{m k}^{\ell}(\beta) N_{\ell}^{k} \int_{0}^{\pi} \sin \theta^{\prime} d \theta^{\prime} \\
& \times e^{2 \pi i f \Delta T \cos \theta^{\prime}} P^{k}\left(\cos \theta^{\prime}\right) \\
& \times \int_{0}^{2 \pi} d \phi^{\prime} e^{i k \phi^{\prime}} \hat{\Omega}_{p^{\prime}} \hat{\Omega}_{q^{\prime}} \hat{\Omega}_{r^{\prime}} \hat{\Omega}_{s^{\prime}}
\end{aligned}
$$

In this integral, we have explicitly indicated all of the dependence on $\alpha$. Notice that while $d_{i}^{a b}$ is a function of $\alpha$, in computational coordinates $d_{i}^{a^{\prime} b^{\prime}}(\beta)$ is independent of $\alpha$ and depends only upon $\beta$. Likewise, the tensor $\Theta_{a^{\prime} b^{\prime} c^{\prime} d^{\prime}}^{p^{\prime} q^{\prime} r^{\prime} s^{\prime}}$ defined by Eq. (5.9) has constant components in the computational frame. Hence the integral over $\alpha$ give a factor $2 \pi$ :

$$
\begin{aligned}
\gamma_{\ell m}(f)= & \frac{5}{8 \pi} d_{1}^{a^{\prime} b^{\prime}}(\beta) d_{2}^{c^{\prime} d^{\prime}}(\beta) \\
& \times \Theta_{a^{\prime} b^{\prime} c^{\prime} d^{\prime}}^{p^{\prime} q^{\prime} r^{\prime} s^{\prime}} \sum_{k=-\ell}^{\ell} d_{m k}^{\ell}(\beta) N_{\ell}^{k} \int_{0}^{\pi} \sin \theta^{\prime} d \theta^{\prime} \\
& \times e^{2 \pi i f \Delta T \cos \theta^{\prime}} P^{k}\left(\cos \theta^{\prime}\right) \\
& \times \int_{0}^{2 \pi} d \phi^{\prime} e^{i k \phi^{\prime}} \hat{\Omega}_{p^{\prime}} \hat{\Omega}_{q^{\prime}} \hat{\Omega}_{r^{\prime}} \hat{\Omega}_{s^{\prime}} .
\end{aligned}
$$

The form of this integral is interesting. The integral over $\phi^{\prime}$ will vanish unless $k=-4, \ldots, 4$, in which case it yields a product of at most four factors of $\sin \theta^{\prime}$ and $\cos \theta^{\prime}$. Introducing a new variable $u=\cos \theta^{\prime}$ the two integrals appearing in Eq. (8.1) may be expressed as linear combinations of the integrals

$$
\int_{-1}^{1} d u e^{2 \pi i f \Delta T u} P^{k}(u) u^{N}\left(1-u^{2}\right)^{|k| / 2}
$$

where $N$ is a non-negative integer bounded by $N+|k| \leqslant 4$. Such integrals can be expressed in closed form as we show in the Appendix.

From this point on, we consider only the case of the two LIGO detectors. In this case the simplest way to proceed is to compute

$$
\begin{aligned}
s_{k}\left(\theta^{\prime}\right)= & d_{1}^{a^{\prime} b^{\prime}}(\beta) d_{2}^{c^{\prime} d^{\prime}}(\beta) \Theta_{a^{\prime} b^{\prime} c^{\prime} d^{\prime}}^{p^{\prime} q^{\prime} r^{\prime} s^{\prime}} \\
& \times \int_{0}^{2 \pi} d \phi^{\prime} e^{i k \phi^{\prime}} \hat{\Omega}_{p^{\prime}} \hat{\Omega}_{q^{\prime}} \hat{\Omega}_{r^{\prime}} \hat{\Omega}_{s^{\prime}}
\end{aligned}
$$

To evaluate this, we use definition (4.5) of the $d_{i}^{a b}$, the contraction operator (5.9), and the arm directions (6.6). Substituting in the vector $\hat{\Omega}^{a^{\prime}}$ given by Eq. (7.11) gives elementary integrals over $\phi^{\prime}$. The results are easily written in terms of the variable $u=\cos \theta^{\prime}$ :

$$
\begin{aligned}
& s_{0}(u)=-3.01308+1.75421 u^{2}+0.945109 u^{4}, \\
& s_{1}(u)= {[0.5451+0.543353 i} \\
&\left.-(1.6954+1.73284 i) u^{2}\right] u\left(1-u^{2}\right)^{1 / 2}, \\
& s_{2}(u)= {[-0.0428705+1.41125 i} \\
&\left.+(0.0119604-0.670812 i) u^{2}\right]\left(1-u^{2}\right), \\
& s_{3}(u)=(-0.245744+0.227815 i) u\left(1-u^{2}\right)^{3 / 2}, \\
& s_{4}(u)=(0.0492709+0.00345516 i)\left(1-u^{2}\right)^{2}, \\
& s_{-k}(u)=s_{k}^{*}(u), \\
& s_{k}(u)=0 \quad \text { for }|k|>4 .
\end{aligned}
$$

We then have

$$
\begin{aligned}
\gamma_{\ell m}(f)= & \frac{5}{8 \pi} \sum_{k=-\ell}^{\ell} d_{m k}^{\ell}(\beta) N_{\ell}^{k} \\
& \times \int_{-1}^{1} d u e^{2 \pi i f \Delta T u} \mathrm{P}^{k}(u) s_{k}(u) .
\end{aligned}
$$

We now evaluate these functions for the first few multipoles. For this purpose we introduce a dimensionless frequency variable $x=2 \pi f \Delta T$. Because $\gamma_{\ell m}=(-1)^{\ell+m} \gamma_{\ell,-m}^{*}$ we give these functions only for $m=0, \ldots, \ell$. They may be conveniently written in terms of spherical Bessel functions $j_{n}$. For $\ell=0$ one has 


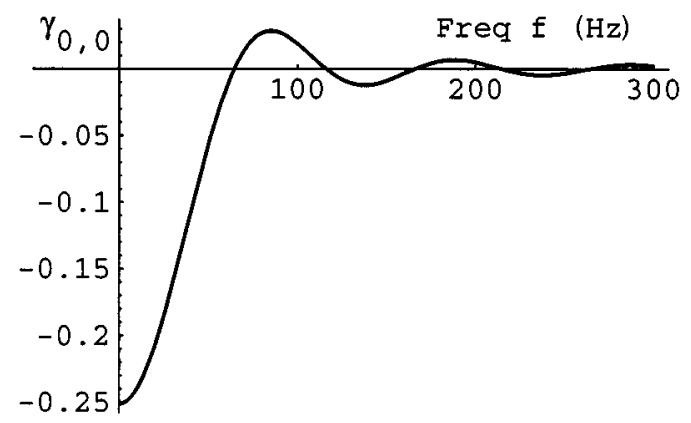

FIG. 1. The (real) function $\gamma_{0,0}(f)$ is shown for the LIGO pair of detectors.

$$
\begin{aligned}
\gamma_{0,0}(x)= & -0.0352174 j_{0}(x)-0.818115 \frac{j_{1}(x)}{x} \\
& +0.848647 \frac{j_{2}(x)}{x^{2}} .
\end{aligned}
$$

A graph of this function is shown in Fig. 1.

For $\ell=1$ one has

$$
\begin{gathered}
\gamma_{1,0}(x)=-0.0279637 i j_{1}(x)-0.252119 i \frac{j_{2}(x)}{x} \\
-1.66955 i \frac{j_{3}(x)}{x^{2}}, \\
\gamma_{1,1}(x)=0.0383329 i j_{1}(x)-(0.327033-1.03547 i) \frac{j_{2}(x)}{x} \\
+(1.90568-1.77847 i) \frac{j_{3}(x)}{x^{2}} .
\end{gathered}
$$

A graph of these functions is shown in Fig. 2.

For $\ell=2$ one has

$$
\begin{aligned}
\gamma_{2,0}(x)= & 0.0145494 j_{0}(x)+1.00009 \frac{j_{1}(x)}{x}-9.39901 \frac{j_{2}(x)}{x^{2}} \\
& +28.0344 \frac{j_{3}(x)}{x^{3}}, \\
\gamma_{2,1}(x)= & 0.0392947 j_{0}(x)+(0.385015+0.335238 i) \frac{j_{1}(x)}{x} \\
& -(2.38288+2.48534 i) \frac{j_{2}(x)}{x^{2}} \\
& -(6.01443-16.1191 i) \frac{j_{3}(x)}{x^{3}}
\end{aligned}
$$

$$
\begin{aligned}
\gamma_{2,2}(x)= & -0.0380887 j_{0}(x)-(1.05867+0.649899 i) \frac{j_{1}(x)}{x} \\
& +(9.05483+6.80403 i) \frac{j_{2}(x)}{x^{2}} \\
& -(18.5056+20.4538 i) \frac{j_{3}(x)}{x^{3}} .
\end{aligned}
$$

A graph of these functions is shown in Fig. 3 .

For $\ell=3$ one has

$$
\begin{aligned}
\gamma_{3,0}(x)= & 0.0416301 i j_{1}(x)+0.805209 i \frac{j_{2}(x)}{x} \\
& -11.2779 i \frac{j_{3}(x)}{x^{2}}+18.399 i \frac{j_{4}(x)}{x^{3}}, \\
\gamma_{3,1}(x)= & 0.00182185 i j_{1}(x)-(0.0155429+1.03107 i) \frac{j_{2}(x)}{x} \\
& -(1.38339-11.1549 i) \frac{j_{3}(x)}{x^{2}}-(7.72558 \\
& +45.6531 i) \frac{j_{4}(x)}{x^{3}}, \\
& +(11.3339-13.8287 i) \frac{j_{3}(x)}{x^{2}} \\
\gamma_{3,2}(x)= & 0.0461979 i j_{1}(x)+(0.788263-0.534988 i) \frac{j_{2}(x)}{x} \\
+ & (7.13839-8.50643 i) \frac{j_{3}(x)}{x^{2}} \\
+ & (42.9458-1.80521 i) \frac{j_{4}(x)}{x^{3}},
\end{aligned}
$$

A graph of these functions is shown in Fig. 4.

For $\ell=4$ one has
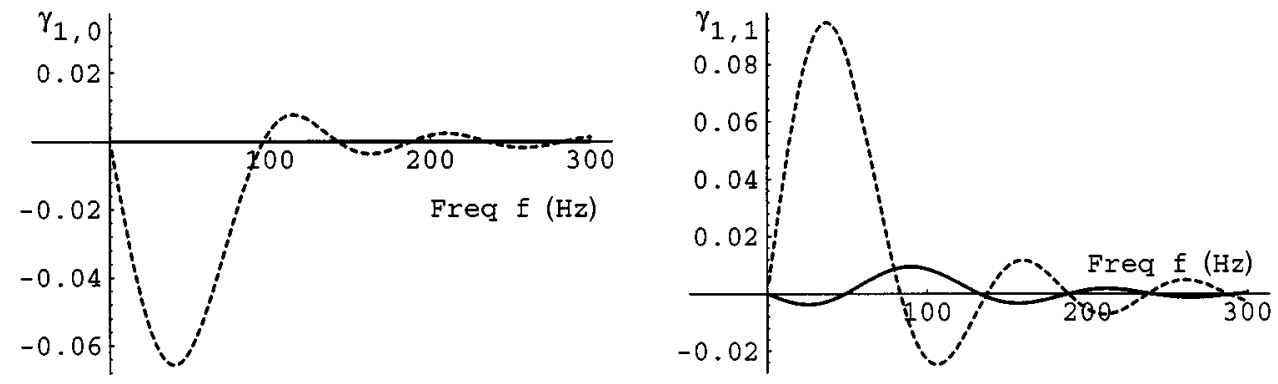

FIG. 2. The functions $\gamma_{1, m}(f)$ are shown for the LIGO pair of detectors. The real parts are shown as the solid curves and the imaginary parts as the dotted curves. 

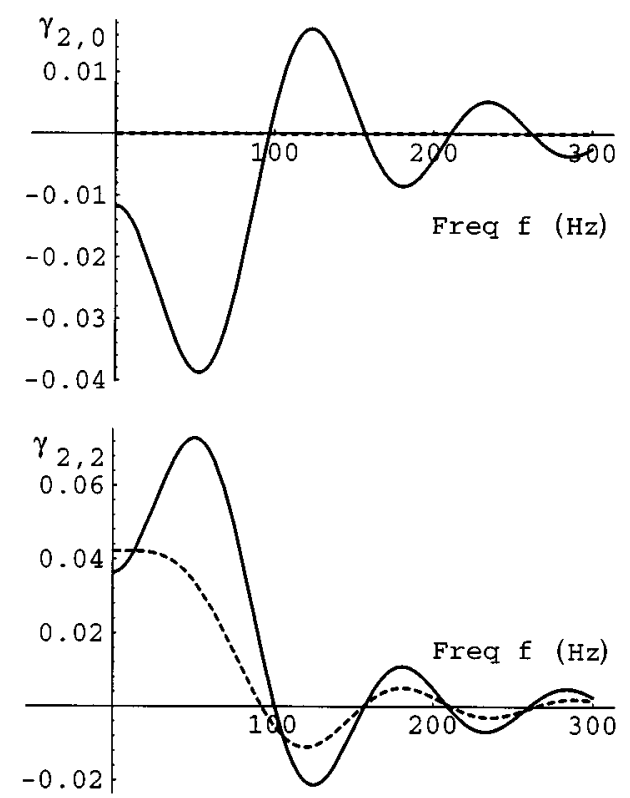

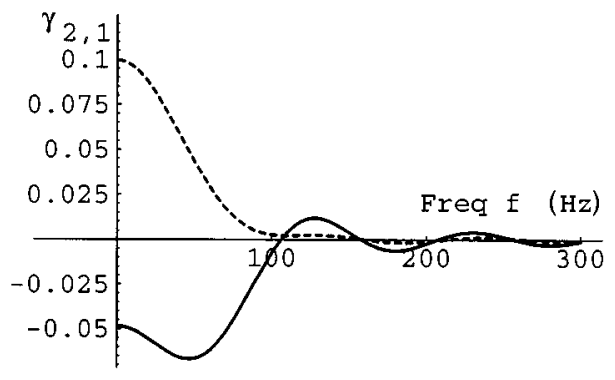

FIG. 3. The functions $\gamma_{2, m}(f)$ are shown for the LIGO pair of detectors. The real parts are shown as the solid curves and the imaginary parts as the dotted curves.

$$
\begin{array}{rlr}
\gamma_{4,0}(x)= & 0.0232299 j_{0}(x)-0.899008 \frac{j_{1}(x)}{x}+9.78424 \frac{j_{2}(x)}{x^{2}} \quad \gamma_{4,2}(x)= & -0.0155407 j_{0}(x)+(1.05639-0.265167 i) \frac{j_{1}(x)}{x} \\
& -88.696 \frac{j_{3}(x)}{x^{3}}+438.555 \frac{j_{4}(x)}{x^{4}}, & -(14.276-0.635514 i) \frac{j_{2}(x)}{x^{2}}+(127.588 \\
\gamma_{4,1}(x)= & -0.0367891 j_{0}(x)-(1.10932+0.313862 i) \frac{j_{1}(x)}{x} & -28.9739 i) \frac{j_{3}(x)}{x^{3}}-(551.647-310.656 i) \frac{j_{4}(x)}{x^{4}}, \\
& +(27.3542+5.98172 i) \frac{j_{2}(x)}{x^{2}}-(204.205 \quad & -(20.3464+20.2033 i) \frac{j_{2}(x)}{x^{2}}+(135.553 \\
& +48.3679 i) \frac{j_{3}(x)}{x^{3}}+(496.286+149.558 i) \frac{j_{4}(x)}{x^{4}}, & +177.464 i) \frac{j_{3}(x)}{x^{3}}-(156.429+719.091 i) \frac{j_{4}(x)}{x^{4}},
\end{array}
$$
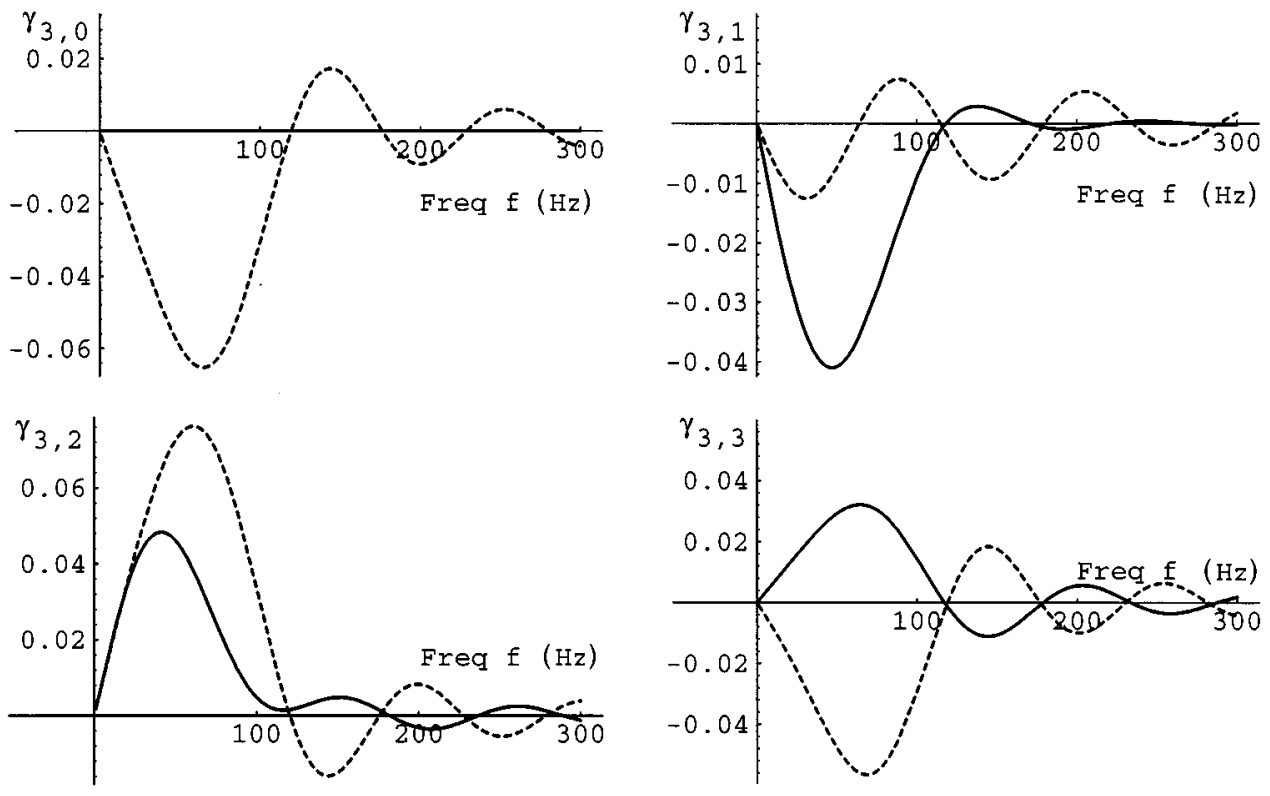

FIG. 4. The functions $\gamma_{3, m}(f)$ are shown for the LIGO pair of detectors. The real parts are shown as the solid curves and the imaginary parts as the dotted curves. 


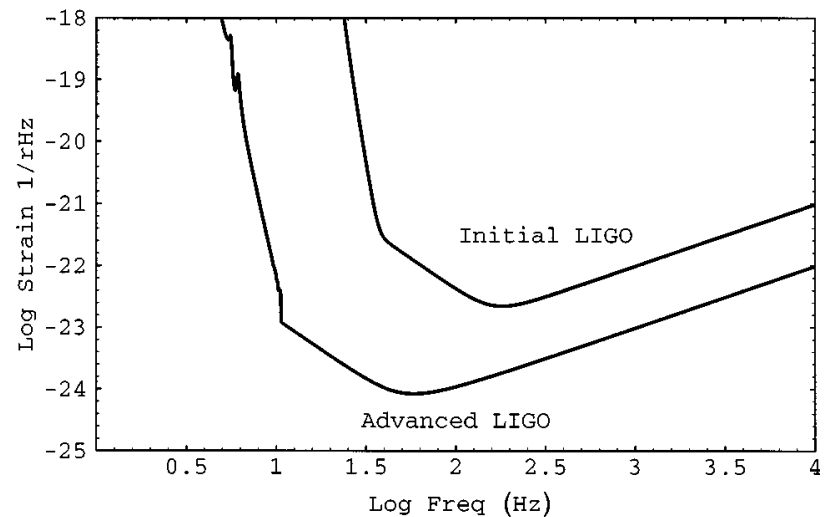

FIG. 5. The predicted noise power spectra of the initial and advanced LIGO detectors. The horizontal axis is $\log _{10}$ of frequency $f$, in Hz. The vertical axis shows $\log _{10}[P(f) / \mathrm{sec}]^{1 / 2}$, or strain per root Hz. These noise power spectra are the published design goals. The bumps appearing in the low-frequency part of the advanced LIGO noise curve are obtained by folding measured seismic noise data with the predicted transfer function of the seismic isolation (stack) system.

$$
\begin{aligned}
\gamma_{4,4}(x)= & -0.0344655 j_{0}(x)-(0.977416+1.17615 i) \frac{j_{1}(x)}{x} \\
& +(24.322+22.0573 i) \frac{j_{2}(x)}{x^{2}}-(183.738 \\
& +163.238 i) \frac{j_{3}(x)}{x^{3}}+(458.028+438.49 i) \frac{j_{4}(x)}{x^{4}}
\end{aligned}
$$

Note that the $\gamma_{\ell m}$ with $\ell$ odd vanish as $f \rightarrow 0$, in contrast with the functions with $\ell$ even, which approach constant values at zero frequency.

\section{OBSERVABILITY AND SIGNAL-TO-NOISE RATIOS}

Up to this point, we have shown how anisotropies in the gravitational-wave stochastic background give rise to periodic variations in the "signal" obtained by correlating a pair of detectors. These periodic variations are described by the Fourier series (5.1), with coefficients $S_{m}$. In this section, we address the question: how precisely can the values of these coefficients be determined, in the presence of noise in the two detectors? We answer this question by calculating the signal-to-noise $(S / N)$ ratios that would arise in measurements of the $S_{m}$, which also permits us to determine the best choice of the optimal filter functions $\widetilde{Q}(f)$.

In carrying out this analysis, we follow the technique used in Sec. 3.2 of Ref. [8]. The following presentation will be somewhat cryptic as we will assume that the reader is familiar with that material.

The noise in the detectors is characterized by a crosscorrelation function

$$
\left\langle\widetilde{n_{i}^{*}}(f) \widetilde{n_{j}}\left(f^{\prime}\right)\right\rangle=\frac{1}{2} \delta_{i j} \delta\left(f-f^{\prime}\right) P_{i}(|f|) .
$$

Here $i=1,2$ labels the two detector sites and $P_{i}(f)$ is the (one-sided, real) noise power spectrum of the $i$ th detector. For the initial and advanced LIGO detectors, these power spectra are shown in Fig. 5. For our calculations of $S / N$ we will need to know the noise properties of the detectors averaged over our "windowing time", $\tau$. To obtain these, we first characterize the detector noise in the time domain, by Fourier transforming Eq. (9.1). This gives

$$
\begin{aligned}
& \left\langle n_{i}(t) n_{j}\left(t^{\prime}\right)\right\rangle \\
& \quad=\int_{-\infty}^{\infty} d f e^{-2 \pi i f t} \int_{-\infty}^{\infty} d f^{\prime} e^{2 \pi i f^{\prime} t^{\prime}}\left\langle\tilde{n}_{i}^{*}(f) \tilde{n}_{j}\left(f^{\prime}\right)\right\rangle \\
& \quad=\frac{1}{2} \delta_{i j} \int_{-\infty}^{\infty} d f e^{-2 \pi i f\left(t-t^{\prime}\right)} P_{i}(|f|) .
\end{aligned}
$$

In words, this says that the Fourier transform of the noise auto-correlation function is the noise power spectrum.

We can now find the "windowed" version of these formulas, using the definition (4.6) of the windowed transform. Taking the windowed transforms of Eq. (9.2) yields

$$
\begin{aligned}
\left\langle\widetilde{n}_{i}^{*}(f, t) \tilde{n}_{j}\left(f^{\prime}, t^{\prime}\right)\right\rangle & \\
= & \int_{t-\tau / 2}^{t+\tau / 2} d t^{\prime \prime} e^{2 \pi i f t^{\prime \prime}} \\
& \times \int_{t^{\prime}-\tau / 2}^{t^{\prime}+\tau / 2} d t^{\prime \prime \prime} e^{-2 \pi i f^{\prime} t^{\prime \prime \prime}}\left\langle n_{i}\left(t^{\prime \prime}\right) n_{j}\left(t^{\prime \prime \prime}\right)\right\rangle \\
= & \frac{1}{2} \delta_{i j} e^{2 \pi i\left(f t-f^{\prime} t^{\prime}\right)} \int_{-\infty}^{\infty} d p e^{-2 \pi i p\left(t-t^{\prime}\right)} \delta_{\tau}(f-p) \\
& \times \delta_{\tau}\left(f^{\prime}-p\right) P_{i}(|p|) .
\end{aligned}
$$

Note that in the long averaging time limit $\tau \rightarrow \infty$ this reproduces Eq. (9.1).

In virtually any realistic scenario, the intrinsic instrumental detector noise is expected to be much larger than the strain arising from the stochastic background of gravity waves. For this reason, if we define the "noise", in a given measurement of $S_{m}$ by $N_{m} \equiv S_{m}-\left\langle S_{m}\right\rangle$ then to good approximation a formula for $N_{m}$ may be obtained by replacing the total detector output $s_{i}(t)$ which appears in Eq. (4.2) by $n_{i}(t)$. Using the definition of the "signal" (4.9) and the definition of the $m$ th harmonic (5.2) one obtains

$$
N_{m}=\frac{1}{T} \int_{0}^{T} d t e^{-i m \omega_{\mathrm{e}} t} \int_{-\infty}^{\infty} d f \widetilde{n}_{1}^{*}(f, t) \widetilde{n_{2}}(f, t) \widetilde{Q}(f) .
$$

(Note that in this formula, we assume that the total observation time $T$ is large compared with Earth's rotation time $T_{\mathrm{e}}$ and that during the observation time the Earth has made an integral number of rotations, so that $T / T_{\mathrm{e}}$ is a large integer. We will typically take $T$ to be 1 sidereal year.) The approximation that we make here is obviously consistent with $\left\langle N_{m}\right\rangle=0$ since the noise in the two detectors is assumed to be uncorrelated, so that $\left\langle\widetilde{n}_{1}^{*}(f, t){\widetilde{n_{2}}}_{2}\left(f^{\prime}, t^{\prime}\right)\right\rangle=0$.

The "noise" arising in the measurement of $S_{m}$ may be characterized by $\left\langle\left|N_{m}\right|^{2}\right\rangle=\left\langle\left|S_{m}\right|^{2}\right\rangle-\left|\left\langle S_{m}\right\rangle\right|^{2}$. We now calculate $\left\langle N_{m}^{*} N_{m^{\prime}}\right\rangle$. Substituting Eq. (9.3) into Eq. (9.4) and "squaring" yields 


$$
\begin{aligned}
\left\langle N_{m}^{*} N_{m^{\prime}}\right\rangle= & \frac{1}{T^{2}} \int_{0}^{T} d t \int_{0}^{T} d t^{\prime} e^{i \omega_{\mathrm{e}}\left(m t-m^{\prime} t^{\prime}\right)} \int_{-\infty}^{\infty} d f \widetilde{Q}^{*}(f) \int_{-\infty}^{\infty} d f^{\prime} \widetilde{Q}\left(f^{\prime}\right)\left\langle\widetilde{n}_{1}^{*}(f, t) \widetilde{n}_{1}\left(f^{\prime}, t^{\prime}\right)\right\rangle\left\langle\widetilde{n}_{2}^{*}(f, t) \widetilde{n_{2}}\left(f^{\prime}, t^{\prime}\right)\right\rangle \\
= & \frac{1}{4 T^{2}} \int_{0}^{T} d t \int_{0}^{T} d t^{\prime} \int_{-\infty}^{\infty} d f \int_{-\infty}^{\infty} d f^{\prime} \int_{-\infty}^{\infty} d p \int_{-\infty}^{\infty} d p^{\prime} \widetilde{Q^{*}}(f) \widetilde{Q}\left(f^{\prime}\right) e^{i \omega_{e}\left(m t-m^{\prime} t^{\prime}\right)+2 \pi i\left(p-p^{\prime}\right)\left(t-t^{\prime}\right)} \delta_{\tau}(f-p) \\
& \times \delta_{\tau}\left(f^{\prime}-p\right) \delta_{\tau}\left(f-p^{\prime}\right) \delta_{\tau}\left(f^{\prime}-p^{\prime}\right) P_{1}(|p|) P_{2}\left(\left|p^{\prime}\right|\right)
\end{aligned}
$$

We can simplify this expression to the point where it is useful, however, this is somewhat tricky-there is only one order in which the integrals above can be simply evaluated to yield useful approximations. We first do the integrals with respect to $t$ and $t^{\prime}$ exactly. This gives

$$
\begin{aligned}
& \left\langle N_{m}^{*} N_{m^{\prime}}\right\rangle \\
& =\frac{1}{4 T^{2}} \int_{-\infty}^{\infty} d f \int_{-\infty}^{\infty} d f^{\prime} \int_{-\infty}^{\infty} d p \int_{-\infty}^{\infty} \\
& \quad \times d p^{\prime} \widetilde{Q}^{*}(f) \widetilde{Q}\left(f^{\prime}\right) P_{1}(|p|) P_{2}\left(\left|p^{\prime}\right|\right) \\
& \quad \times \exp \left[-i \omega_{\mathrm{e}} T\left(m-m^{\prime}\right) / 2\right] \delta_{T}\left(p-p^{\prime}+m \omega_{\mathrm{e}} / 2 \pi\right) \\
& \quad \times \delta_{T}\left(p-p^{\prime}+m^{\prime} \omega_{\mathrm{e}} / 2 \pi\right) \delta_{\tau}(f-p) \delta_{\tau}\left(f^{\prime}-p\right) \\
& \quad \times \delta_{\tau}\left(f-p^{\prime}\right) \delta_{\tau}\left(f^{\prime}-p^{\prime}\right)
\end{aligned}
$$

Now we note that the effective support of $\delta_{T}(f)$ extends over a very narrow range of frequencies (typically, $|f|<10^{-7} \mathrm{~Hz}$ ) compared with the effective support of $\delta_{\tau}(f)$ (whose support is typically $|f|<10^{-2} \mathrm{~Hz}$ ). In addition, none of the remaining integrand varies over such a narrow frequency range. So we are justified in replacing $\delta_{T}\left(p-p^{\prime}+m \omega_{\mathrm{e}} / 2 \pi\right)$ by the ordinary Dirac delta function $\delta\left(p-p^{\prime}+m \omega_{\mathrm{e}} / 2 \pi\right)$. This gives

$$
\begin{aligned}
\left\langle N_{m}^{*} N_{m^{\prime}}\right\rangle & \\
= & \frac{1}{4 T^{2}} \int_{-\infty}^{\infty} d f \int_{-\infty}^{\infty} d f^{\prime} \widetilde{Q}^{*}(f) \widetilde{Q}\left(f^{\prime}\right) \\
& \times \int_{-\infty}^{\infty} d p^{\prime} \delta_{\tau}\left(f-p^{\prime}\right) \delta_{\tau}\left(f^{\prime}-p^{\prime}\right) \\
& \times \exp \left[-i \omega_{\mathrm{e}} T\left(m-m^{\prime}\right) / 2\right] \delta_{T}\left(\frac{\omega_{\mathrm{e}}\left(m-m^{\prime}\right)}{2 \pi}\right) \\
& \times \delta_{\tau}\left(f-p^{\prime}+\frac{m \omega_{\mathrm{e}}}{2 \pi}\right) \delta_{\tau}\left(f^{\prime}-p^{\prime}+\frac{m \omega_{\mathrm{e}}}{2 \pi}\right) \\
& \times P_{1}\left(\left|p^{\prime}-\frac{m \omega_{\mathrm{e}}}{2 \pi}\right|\right) P_{2}\left(\left|p^{\prime}\right|\right) .
\end{aligned}
$$

If we now additionally assume that the observation time $T$ is much greater than the period of a single rotation $T_{\mathrm{e}}=2 \pi / \omega_{\mathrm{e}}$ then to good approximation $\delta_{T}\left(\omega_{\mathrm{e}}\left(m-m^{\prime}\right) / 2 \pi\right) \approx T \delta_{m m^{\prime}}$. Thus

$$
\begin{aligned}
& \left\langle N_{m}^{*} N_{m^{\prime}}\right\rangle \\
& =\frac{1}{4 T} \delta_{m m^{\prime}} \int_{-\infty}^{\infty} d f \int_{-\infty}^{\infty} d f^{\prime} \widetilde{Q}^{*}(f) \widetilde{Q}\left(f^{\prime}\right) \\
& \quad \times \int_{-\infty}^{\infty} d p \delta_{\tau}(f-p) \delta_{\tau}\left(f^{\prime}-p\right) \delta_{\tau}\left(f-p+\frac{m \omega_{\mathrm{e}}}{2 \pi}\right) \\
& \quad \times \delta_{\tau}\left(f^{\prime}-p+\frac{m \omega_{\mathrm{e}}}{2 \pi}\right) P_{1}\left(\left|p-\frac{m \omega_{\mathrm{e}}}{2 \pi}\right|\right) \\
& \quad \times P_{2}(|p|) .
\end{aligned}
$$

Next we note that the width of the $\delta_{\tau}(f)$ in frequency space is quite large compared to $m \omega_{\mathrm{e}}$ provided that we restrict $m$ to be fairly small: $|m|<T_{\mathrm{e}} / \tau$. We also assume that the noise power spectrum $P_{1}(f)$ does not vary significantly over frequency scales of $\omega_{\mathrm{e}} \approx 10^{-5} \mathrm{~Hz}$. In this case we can neglect the shifting of the arguments by $m \omega_{\mathrm{e}}$ above, obtaining

$$
\begin{aligned}
\left\langle N_{m}^{*} N_{m^{\prime}}\right\rangle= & \frac{1}{4} \frac{\tau}{T} \delta_{m m^{\prime}} \int_{-\infty}^{\infty} d f \int_{-\infty}^{\infty} d f^{\prime} \widetilde{Q}^{*}(f) \widetilde{Q}\left(f^{\prime}\right) \\
& \times \delta_{\tau}^{2}\left(f-f^{\prime}\right) P_{1}(|f|) P_{2}(f \mid) \\
= & \delta_{m m^{\prime}} \frac{\tau^{2}}{4 T} \int_{-\infty}^{\infty} d f|\widetilde{Q}(f)|^{2} P_{1}(|f|) P_{2}(|f|)
\end{aligned}
$$

Setting $m=m^{\prime}$ we finally obtain an expression for the expected "squared noise" in a measurement of $S_{m}$ :

$$
\left\langle\left|N_{m}\right|^{2}\right\rangle=\frac{\tau^{2}}{4 T} \int_{-\infty}^{\infty} d f|\widetilde{Q}(f)|^{2} P_{1}(|f|) P_{2}(|f|)
$$

We can make use of this expression to find the optimal filter $\widetilde{Q}(f)$.

The (squared) signal-to-noise ratio $S / N$ for the $m$ th harmonic is now defined via the ratio of expected signal (magnitude squared) divided by expected (squared) noise. Making use of Eq. (5.3) for the former quantity, and Eq. (9.10) for the latter yields 


$$
\begin{aligned}
\left(\frac{S}{N}\right)_{m}^{2} & \equiv \frac{\left|\left\langle S_{m}\right\rangle\right|^{2}}{\left\langle\left|N_{m}\right|^{2}\right\rangle} \\
& =\frac{4 T(8 \pi / 5)^{2}\left|\tau \int_{-\infty}^{\infty} d f \widetilde{Q}(f) H(f) \sum_{\ell=|m|}^{\infty} p_{\ell m} \gamma_{\ell m}(f)\right|^{2}}{\tau^{2} \int_{-\infty}^{\infty} d f|\widetilde{Q}(f)|^{2} P_{1}(|f|) P_{2}(|f|)}
\end{aligned}
$$

Notice that the averaging time $\tau$ (which was earlier chosen in a rather arbitrary manner) drops out of this expression. Pro- vided that the assumptions about $\tau$ (4.1) used in deriving this equation are satisfied, the actual value is irrelevant.

In order to find the optimal filter function, it is useful to introduce an inner product. For any complex functions of frequency $A(f)$ and $B(f)$, this defines a complex number which is denoted by $(A, B)$. The definition is

$$
(A, B) \equiv \int_{-\infty}^{\infty} d f A^{*}(f) B(f) P_{1}(|f|) P_{2}(|f|) .
$$

This inner product is positive definite because $(A, A)$ is real and non-negative, vanishing only if $A$ is zero. In terms of this inner product, the signal-to-noise ratio may be written as

$$
\left(\frac{S}{N}\right)_{m}^{2}=4 T\left(\frac{8 \pi}{5}\right)^{2} \frac{\left|\left(\widetilde{Q}, H(f) / P_{1}(|f|) P_{2}(|f|) \sum_{\ell=|m|}^{\infty} p_{/ m}^{*} \gamma_{/ m}^{*}(f)\right)\right|^{2}}{(\widetilde{Q}, \widetilde{Q})} .
$$

The optimal choice of filter function $Q(f)$ for determining the $m$ th harmonic is the one which maximizes this ratio. The largest value is obtained by choosing

$$
\widetilde{Q}_{m}(f)=\frac{H(f)}{P_{1}(|f|) P_{2}(|f|)} \sum_{\ell=|m|}^{\infty} p_{\ell m}^{*} \gamma_{/ m}^{*}(f) .
$$

Using the definition (3.11) of $H(f)$ in terms of the spectral function $\Omega_{\mathrm{GW}}(f)$, and substituting the optimal filter $\widetilde{Q}_{m}$ into the expression for $S / N$ yields

$$
\begin{aligned}
\left(\frac{S}{N}\right)_{m}^{2}= & \frac{9 H_{0}^{4}}{50 \pi^{4}} T \int_{0}^{\infty} d f \frac{\Omega_{\mathrm{GW}}^{2}(f)}{f^{6} P_{1}(f) P_{2}(f)} \\
& \times\left|\sum_{\ell=m}^{\infty} p_{\ell m} \gamma_{\ell m}(f)\right|^{2} .
\end{aligned}
$$

For any given source of stochastic gravitational waves, one can use this formula for $(S / N)_{m}$ to determine the observation time $T$ required to observe the $m$ th harmonic of the signal as the Earth rotates relative to the cosmological frame. In precise analogy with the analysis given in [8], the $m$ th harmonic is observable with $90 \%$ confidence if $(S / N)_{m}$ exceeds 1.65 .

\section{EXAMPLE: DIPOLE INDUCED BY PROPER MOTION}

It is well known that the electromagnetic background radiation, generally referred to as the cosmic microwave back- ground radiation (CMBR), is highly isotropic. The largest deviation from isotropy results from the motion of our local system (the solar system barycenter) with respect to the cosmological rest frame. Analysis of data from the Cosmic Background Explorer (COBE) satellite shows that our local system is moving with a velocity $\beta_{\text {proper }} \equiv v / c=0.001236$ in the direction $\left(l=264^{\circ}, b=48^{\circ}\right)$ in galactic coordinates, or equivalently $\left(\alpha=168^{\circ}, \delta=-7^{\circ}\right)$ in celestial coordinates [12]. To lowest order in our proper velocity, this gives rise to an anisotropy in the CMBR described by the temperature distribution

$$
T(\gamma)=T_{0}\left(1+\beta_{\text {proper }} \cos \gamma\right),
$$

where $\gamma$ is the angle between a point on the sky and the velocity vector of our local system, and $T_{0}=2.73 \mathrm{~K}$ is the mean temperature of the CMBR.

In this section, we address the question: would a corresponding dipole moment in the stochastic gravity wave background, arising from the proper motion of the solar system barycenter, be observable with either the initial or advanced LIGO detectors? In the previous section, we calculated the signal-to-noise ratio for observations of the $m$ th harmonic $S_{m}$ (with respect to Earth's rotation) of the signal (obtained by correlating two gravitational-wave detectors). This is determined entirely by the quantities

$$
\begin{gathered}
H_{0}=\text { Hubble expansion rate, } \mathrm{sec}^{-1}, \\
T=\text { Total observation time, sec, } \\
\gamma_{\ell m}(f)=\text { Overlap reduction functions, dimensionless, } \\
\Omega_{\mathrm{GW}}=\text { Fractional energy density in gravitational waves, dimensionless, } \\
p_{\ell m}=\text { Normalized multipoles of GW stochastic background, dimensionless, } \\
P_{i}=\text { Noise power spectral density of detector } i, \text { sec. }
\end{gathered}
$$


As an example, we use this formula to answer the question posed above.

Let us make the following reasonable assumptions: (1) $\Omega_{\mathrm{GW}}(f)$ is constant in the LIGO band and (2) the stochastic gravitational-wave background is isotropic in the same rest frame as the CMBR. The anisotropies in the stochastic background resulting from our proper motion are then described by

$$
\begin{aligned}
& \sum_{\ell m} p_{\ell m} Y_{\ell m}(\bar{\theta}, \bar{\phi}) \\
& =1+\beta_{\text {proper }}\left(\cos \bar{\theta} \cos 97^{\circ}+\sin \bar{\theta} \sin 97^{\circ} \cos \bar{\phi}\right),
\end{aligned}
$$

where the angles above are standard spherical coordinates in the (barred) Earth frame. One thus obtains the following multipole moments:

$$
\begin{gathered}
p_{00}=\sqrt{4 \pi}, \\
p_{1,-1}=\beta_{\text {proper }} \sqrt{\frac{2 \pi}{3}} \sin 97^{\circ}, \\
p_{1,0}=\beta_{\text {proper }} \sqrt{\frac{4 \pi}{3}} \cos 97^{\circ}, \\
p_{1,1}=-\beta_{\text {proper }} \sqrt{\frac{2 \pi}{3}} \sin 97^{\circ}, \\
p_{\ell m}=0 \text { for } \ell>1 .
\end{gathered}
$$

To detect this signal, the optimal filter functions are

$$
Q_{m}(f)=C_{m} \frac{1}{f^{3} P_{1}(|f|) P_{2}(|f|)} \sum_{\ell=|m|}^{\infty} p_{\ell m}^{*} \gamma_{\ell m}^{*},
$$

where $C_{m}$ is an (irrelevant) normalization constant. Making use of the optimal filter functions $Q_{0}$ and $Q_{1}$ for the monopole and dipole terms, we can make predictions about how large $\Omega_{\mathrm{gw}}$ needs to be in order that $S_{0}$ and $S_{1}$ are observable with a given level of confidence in a given observation time.

We can express the sensitivity of a search for the $m$ th harmonic in terms of the minimum value of $\Omega_{\mathrm{GW}}$ necessary to observe it with $90 \%$ confidence. For $90 \%$ confidence we need a signal-to-noise of 1.65 . The minimum value of $\Omega_{\mathrm{GW}}$ is then given for the $m$ th harmonic by

$$
\begin{aligned}
\Omega_{90 \%}^{(m)}= & (1.65) \sqrt{\frac{9}{50}} \frac{H_{0}^{2}}{\pi^{2}} T\left[\int_{0}^{\infty} \frac{d f}{f^{6} P_{1}(f) P_{2}(f)}\right. \\
& \left.\times\left|\sum_{\ell=m \mid}^{\infty} p_{\ell m} \gamma_{\ell m}(f)\right|^{2}\right]^{-1 / 2} .
\end{aligned}
$$

These values are shown in Table I for the initial and advanced LIGO detectors. We note that there are potential sources of sufficient intensity that a dipole might be observable with the advanced LIGO detector. These include stochastic backgrounds due to cosmic strings or to a population of unresolved cosmological-distance supernovae.

\section{FILTER NORMALIZATIONS AND SIGNAL STRENGTHS}

Specific models for an anisotropic background predict signal harmonics $S_{m}$ of definite amplitudes and phases. The phase of a given $S_{m}$ depends upon the chosen origin of time; shifting the origin of time changes the phase of $S_{m}$ but not its amplitude. In order to falsify or confirm a particular anisotropic model, one needs to predict the set of complex numbers $S_{m}$. However, these numbers depend upon the normalization $C_{m}$ of the optimal filter function $Q_{m}(f)$ so in order to make predictions, we need to adopt a convention for these.

We have already chosen optimal filters for which the expected value of $S_{m}$ is real. At least upon first inspection, there do not appear to be any very convenient choices for the overall scale of normalization. Here, for illustrative purposes we adopt (what appears to us as) the least disagreeable of these. We choose the normalization $C_{m}$ so that the expected amplitude of the noise ${ }^{2}$ is unity: $\left\langle\left|N_{m}\right|^{2}\right\rangle=1$. We also assume that $C_{m}$ is positive and real. Note that with this choice, the "signal" is dimensionless. From Eq. (9.10) this gives

$$
C_{m}=2 \sqrt{\frac{T}{\tau}}\left[\int_{-\infty}^{\infty} \frac{d f}{P_{1}(|f|) P_{2}(|f|)}\left|\sum_{\ell=|m|}^{\infty} p_{\ell m} \gamma_{\ell m}(f)\right|^{2}\right]^{-1 / 2} .
$$

Having chosen a normalization, we can list the expected signals for a given detector. If the actual signal value exceeds 1.65 then it has been detected with $90 \%$ confidence. For example, in the case of the dipole source just discussed, the expected signals are given by Eq. (5.3) as

$$
\begin{aligned}
\left\langle S_{m}\right\rangle= & \frac{3 H_{0}^{2}}{10 \pi^{2}} \sqrt{T} \Omega_{\mathrm{GW}}\left[\int_{-\infty}^{\infty} \frac{d f}{P_{1}(|f|) P_{2}(|f|)}\right. \\
& \left.\times\left|\sum_{\ell=|m|}^{\infty} p_{\ell m} \gamma_{\ell m}(f)\right|^{2}\right]^{1 / 2} .
\end{aligned}
$$

Evaluating this for our previous example gives

$$
\left\langle S_{0}\right\rangle=\left\{\begin{array}{l}
1.65 \sqrt{\frac{T}{1 \text { year }}} \frac{\Omega_{\mathrm{GW}} h_{100}^{2}}{1.6 \times 10^{-6}} \quad \text { for initial LIGO, } \\
1.65 \sqrt{\frac{T}{1 \text { year }}} \frac{\Omega_{\mathrm{GW}} h_{100}^{2}}{1.6 \times 10^{-11}} \quad \text { for advanced LIGO, }
\end{array}\right.
$$$$
\left\langle S_{1}\right\rangle=\left\{\begin{array}{l}
1.65 \sqrt{\frac{T}{1 \text { year }}} \frac{\Omega_{\mathrm{GW}} h_{100}^{2}}{2.5 \times 10^{-3}} \text { for initial LIGO, } \\
1.65 \sqrt{\frac{T}{1 \text { year }}} \frac{\Omega_{\mathrm{GW}} h_{100}^{2}}{5.3 \times 10^{-8}} \text { for advanced LIGO, }
\end{array}\right.
$$$$
\left\langle S_{m}\right\rangle=0 \text { for } m \geqslant 2 .
$$

\section{GALACTIC SOURCES}

In this section we consider the possibility of detecting anisotropies in the stochastic gravitational wave background assuming that this background either originates in, or is scattered in the same way as, the luminous matter in our galaxy, and for sources distributed in the same way as the galactic halo. It appears very unlikely that in the LIGO-VIRGO-GEO frequency band there are any sources of a stochastic gravitational-wave background distributed in this way. However, the absence of such harmonics would be one way to 
TABLE I. Sensitivity of initial and advanced LIGO detectors to a dipole term in the gravitational stochastic background, arising from motion of our local system. This table shows the intensity of stochastic background required to detect either the monopole $\left(S_{0}\right)$ or dipole $\left(S_{1}\right)$ term in the signal, with $90 \%$ confidence, in one year of observation.

\begin{tabular}{lcc}
\hline \hline & Initial LIGO & Advanced LIGO \\
\hline Monopole $(m=0)$ & $\Omega_{90 \%}=1.6 \times 10^{-6} h_{100}^{-2}$ & $\Omega_{90 \%}=1.6 \times 10^{-11} h_{100}^{-2}$ \\
Dipole $(m=1)$ & $\Omega_{90 \%}=2.5 \times 10^{-3} h_{100}^{-2}$ & $\Omega_{90 \%}=5.3 \times 10^{-8} h_{100}^{-2}$ \\
\hline \hline
\end{tabular}

demonstrate that a stochastic background had extra-galactic origin.

For the distribution of luminous matter in the galaxy we consider a set of three models constructed by Kent, Dame, and Fazio [13] to model $2.4 \mu \mathrm{m}$ data from the infrared telescope taken as part of the Spacelab 2 mission. These models all assume cylindrical symmetry and take the total luminosity to consist of two components; one, $\nu_{D}(r, z)$, modeling the disk and the other, $\nu_{B}(r, z)$, modeling the central bulge, where $r$ and $z$ denote cylindrical polar coordinates based at the center of the galaxy.

The first model, which we refer to as Kent-Dame-Fazio 1 (KDF1), takes

$$
\begin{gathered}
\nu_{D}(r, z)=\mu_{D} e^{-r / h_{r}} \frac{\operatorname{sech}^{2}\left[z /\left(2 h_{z}\right)\right]}{4 h_{z}}, \\
\nu_{B}(r, z)=\mu_{B} \frac{K_{0}\left(\left\{r^{4}+\left[z /\left(1-\epsilon_{B}\right)\right]^{4}\right\}^{1 / 4} / h_{B}\right)}{\pi h_{B}},
\end{gathered}
$$

where $\mu_{D}=1072 L_{\odot} \mathrm{pc}^{-2}, h_{r}=2775 \mathrm{pc}, h_{z}=121 \mathrm{pc}, \mu_{B}$ $=6208 L_{\odot} \mathrm{pc}^{-2}, h_{B}=634 \mathrm{pc}$, and $\epsilon_{B}=0.26$. Here $K_{0}$ denotes the modified Bessel function of order 0 .

The second model (KDF2) takes

$$
\begin{gathered}
\nu_{D}(r, z)=\mu_{D} e^{-r / h_{r}} \frac{\exp \left[-\left|z /\left(2 h_{z}\right)\right|\right]}{2 h_{z}}, \\
\nu_{B}(r, z)=\mu_{B} \frac{K_{0}\left(\left\{r^{4}+\left[z /\left(1-\epsilon_{B}\right)\right]^{4}\right\}^{1 / 4} / h_{B}\right)}{\pi h_{B}},
\end{gathered}
$$

where $\mu_{D}=1208 L_{\odot} \mathrm{pc}^{-2}, h_{r}=2694 \mathrm{pc}, h_{z}=204 \mathrm{pc}, \mu_{B}$ $=7710 L_{\odot} \mathrm{pc}^{-2}, h_{B}=500 \mathrm{pc}$, and $\epsilon_{B}=0.19$.

The third model (KDF3) takes

$$
\nu_{D}(r, z)=\mu_{D} e^{-r / h_{r}} \frac{\exp \left\{-\left|z /\left[2 h_{z}(r)\right]\right|\right\}}{2 h_{z}(r)},
$$

TABLE II. Sensitivity of the initial LIGO detector to the first 25 multipoles in the gravitational stochastic background, assumed to follow the luminosity of the galaxy or galactic halo. This table shows the intensity of stochastic background required to detect the multipole $S_{m}$ with $90 \%$ confidence in one year of observation.

\begin{tabular}{lcccc}
\hline \hline$m$ & $h_{100}^{2} \Omega_{90 \%} \mathrm{KDF} 1$ & $h_{100^{2} \Omega_{90 \%} \mathrm{KDF} 2}$ & $h_{100}^{2} \Omega_{90 \%} \mathrm{KDF} 3$ & $h_{100^{2} \Omega_{90 \%} \text { halo }}$ \\
\hline 0 & $1.4 \times 10^{-5}$ & $1.4 \times 10^{-5}$ & $1.4 \times 10^{-5}$ & $3.9 \times 10^{-3}$ \\
1 & $1.4 \times 10^{-5}$ & $1.3 \times 10^{-5}$ & $1.3 \times 10^{-5}$ & $3.4 \times 10^{-3}$ \\
2 & $1.9 \times 10^{-5}$ & $1.9 \times 10^{-5}$ & $1.8 \times 10^{-5}$ & $3.6 \times 10^{-3}$ \\
3 & $3.0 \times 10^{-5}$ & $2.9 \times 10^{-5}$ & $2.9 \times 10^{-5}$ & $4.2 \times 10^{-3}$ \\
4 & $4.6 \times 10^{-5}$ & $4.4 \times 10^{-5}$ & $4.6 \times 10^{-5}$ & $5.0 \times 10^{-3}$ \\
5 & $7.3 \times 10^{-5}$ & $6.9 \times 10^{-5}$ & $7.1 \times 10^{-5}$ & $6.1 \times 10^{-3}$ \\
6 & $7.8 \times 10^{-5}$ & $7.4 \times 10^{-5}$ & $7.5 \times 10^{-5}$ & $8.1 \times 10^{-3}$ \\
7 & $8.6 \times 10^{-5}$ & $8.0 \times 10^{-5}$ & $8.3 \times 10^{-5}$ & $1.2 \times 10^{-2}$ \\
8 & $1.2 \times 10^{-4}$ & $1.1 \times 10^{-4}$ & $1.1 \times 10^{-4}$ & $1.7 \times 10^{-2}$ \\
9 & $2.0 \times 10^{-4}$ & $2.0 \times 10^{-4}$ & $1.9 \times 10^{-4}$ & $2.7 \times 10^{-2}$ \\
10 & $3.6 \times 10^{-4}$ & $4.0 \times 10^{-4}$ & $3.5 \times 10^{-4}$ & $4.3 \times 10^{-2}$ \\
11 & $5.0 \times 10^{-4}$ & $5.2 \times 10^{-4}$ & $4.9 \times 10^{-4}$ & $7.0 \times 10^{-2}$ \\
12 & $6.3 \times 10^{-4}$ & $6.2 \times 10^{-4}$ & $6.2 \times 10^{-4}$ & $1.2 \times 10^{-1}$ \\
13 & $9.2 \times 10^{-4}$ & $8.9 \times 10^{-4}$ & $9.0 \times 10^{-4}$ & $2.0 \times 10^{-1}$ \\
14 & $1.5 \times 10^{-3}$ & $1.5 \times 10^{-3}$ & $1.5 \times 10^{-3}$ & $3.1 \times 10^{-1}$ \\
15 & $2.4 \times 10^{-3}$ & $2.5 \times 10^{-3}$ & $2.4 \times 10^{-3}$ & $5.0 \times 10^{-1}$ \\
16 & $3.6 \times 10^{-3}$ & $3.7 \times 10^{-3}$ & $3.6 \times 10^{-3}$ & $7.8 \times 10^{-1}$ \\
17 & $5.1 \times 10^{-3}$ & $5.2 \times 10^{-3}$ & $5.1 \times 10^{-3}$ & $1.2 \times 10^{0}$ \\
18 & $7.3 \times 10^{-3}$ & $7.2 \times 10^{-3}$ & $7.1 \times 10^{-3}$ & $1.7 \times 10^{0}$ \\
19 & $1.0 \times 10^{-2}$ & $1.0 \times 10^{-2}$ & $1.0 \times 10^{-2}$ & $2.4 \times 10^{0}$ \\
20 & $1.4 \times 10^{-2}$ & $1.4 \times 10^{-2}$ & $1.4 \times 10^{-2}$ & $3.6 \times 10^{0}$ \\
21 & $2.1 \times 10^{-2}$ & $2.1 \times 10^{-2}$ & $2.0 \times 10^{-2}$ & $5.0 \times 10^{0}$ \\
22 & $2.9 \times 10^{-2}$ & $2.9 \times 10^{-2}$ & $2.8 \times 10^{-2}$ & $6.5 \times 10^{0}$ \\
23 & $4.0 \times 10^{-2}$ & $3.9 \times 10^{-2}$ & $3.8 \times 10^{-2}$ & $8.9 \times 10^{0}$ \\
24 & $5.1 \times 10^{-2}$ & $5.0 \times 10^{-2}$ & $4.9 \times 10^{-2}$ & $1.2 \times 10^{1}$ \\
\hline \hline & & & & \\
\hline
\end{tabular}


TABLE III. Sensitivity of the advanced LIGO detector to the first 25 multipoles in the gravitational stochastic background, assumed to follow the luminosity of the galaxy or galactic halo. This table shows the intensity of stochastic background required to detect the multipole $S_{m}$ with $90 \%$ confidence in one year of observation.

\begin{tabular}{lcccc}
\hline \hline$m$ & $h_{100}^{2} \Omega_{90 \%} \mathrm{KDF} 1$ & $h_{100}^{2} \Omega_{90 \%} \mathrm{KDF} 2$ & $h_{100}^{2} \Omega_{90 \%} \mathrm{KDF} 3$ & $h_{100}^{2} \Omega_{90 \%}$ halo \\
\hline 0 & $1.8 \times 10^{-10}$ & $1.7 \times 10^{-10}$ & $1.7 \times 10^{-10}$ & $6.7 \times 10^{-8}$ \\
1 & $4.8 \times 10^{-10}$ & $4.7 \times 10^{-10}$ & $4.7 \times 10^{-10}$ & $7.8 \times 10^{-8}$ \\
2 & $1.1 \times 10^{-9}$ & $1.0 \times 10^{-9}$ & $1.0 \times 10^{-9}$ & $1.1 \times 10^{-7}$ \\
3 & $5.3 \times 10^{-9}$ & $5.0 \times 10^{-9}$ & $5.4 \times 10^{-9}$ & $2.6 \times 10^{-7}$ \\
4 & $6.4 \times 10^{-9}$ & $6.0 \times 10^{-9}$ & $6.3 \times 10^{-9}$ & $6.4 \times 10^{-7}$ \\
5 & $1.1 \times 10^{-8}$ & $1.0 \times 10^{-8}$ & $1.0 \times 10^{-8}$ & $1.6 \times 10^{-6}$ \\
6 & $3.1 \times 10^{-8}$ & $3.0 \times 10^{-8}$ & $3.0 \times 10^{-8}$ & $4.4 \times 10^{-6}$ \\
7 & $1.2 \times 10^{-7}$ & $1.3 \times 10^{-7}$ & $1.2 \times 10^{-7}$ & $1.3 \times 10^{-5}$ \\
8 & $2.4 \times 10^{-7}$ & $2.4 \times 10^{-7}$ & $2.4 \times 10^{-7}$ & $2.7 \times 10^{-5}$ \\
9 & $4.5 \times 10^{-7}$ & $4.2 \times 10^{-7}$ & $4.5 \times 10^{-7}$ & $6.3 \times 10^{-5}$ \\
10 & $1.1 \times 10^{-6}$ & $1.0 \times 10^{-6}$ & $1.1 \times 10^{-6}$ & $1.3 \times 10^{-4}$ \\
11 & $3.0 \times 10^{-6}$ & $3.3 \times 10^{-6}$ & $3.1 \times 10^{-6}$ & $2.6 \times 10^{-4}$ \\
12 & $5.5 \times 10^{-6}$ & $5.5 \times 10^{-6}$ & $5.7 \times 10^{-6}$ & $4.5 \times 10^{-4}$ \\
13 & $8.3 \times 10^{-6}$ & $7.7 \times 10^{-6}$ & $8.7 \times 10^{-6}$ & $7.7 \times 10^{-4}$ \\
14 & $1.5 \times 10^{-5}$ & $1.5 \times 10^{-5}$ & $1.6 \times 10^{-5}$ & $1.3 \times 10^{-3}$ \\
15 & $3.0 \times 10^{-5}$ & $3.2 \times 10^{-5}$ & $3.1 \times 10^{-5}$ & $2.0 \times 10^{-3}$ \\
16 & $4.4 \times 10^{-5}$ & $4.4 \times 10^{-5}$ & $4.7 \times 10^{-5}$ & $3.0 \times 10^{-3}$ \\
17 & $6.0 \times 10^{-5}$ & $5.6 \times 10^{-5}$ & $6.4 \times 10^{-5}$ & $4.4 \times 10^{-3}$ \\
18 & $9.4 \times 10^{-5}$ & $9.0 \times 10^{-5}$ & $1.0 \times 10^{-4}$ & $6.4 \times 10^{-3}$ \\
19 & $1.6 \times 10^{-4}$ & $1.6 \times 10^{-4}$ & $1.7 \times 10^{-4}$ & $9.0 \times 10^{-3}$ \\
20 & $2.1 \times 10^{-4}$ & $2.1 \times 10^{-4}$ & $2.3 \times 10^{-4}$ & $1.2 \times 10^{-2}$ \\
21 & $2.7 \times 10^{-4}$ & $2.5 \times 10^{-4}$ & $3.0 \times 10^{-4}$ & $1.7 \times 10^{-2}$ \\
22 & $3.9 \times 10^{-4}$ & $3.7 \times 10^{-4}$ & $4.2 \times 10^{-4}$ & $2.3 \times 10^{-2}$ \\
23 & $5.9 \times 10^{-4}$ & $5.8 \times 10^{-4}$ & $6.4 \times 10^{-4}$ & $3.0 \times 10^{-2}$ \\
24 & $7.8 \times 10^{-4}$ & $7.5 \times 10^{-4}$ & $8.4 \times 10^{-4}$ & $3.9 \times 10^{-2}$ \\
\hline \hline & & & &
\end{tabular}

$$
\nu_{B}(r, z)=\mu_{B} \frac{K_{0}\left(\left\{r^{4}+\left[z /\left(1-\epsilon_{B}\right)\right]^{4}\right\}^{1 / 4} / h_{B}\right)}{\pi h_{B}},
$$

where $\quad \mu_{D}=978 L_{\odot} \mathrm{pc}^{-2}, h_{r}=3001 \mathrm{pc}, \quad \mu_{B}=7395 L_{\odot}$ $\mathrm{pc}^{-2}, h_{B}=667 \mathrm{pc}, \epsilon_{B}=0.39$, and $h_{z}(r)$ is now a function given by

$$
h_{z}(r)=\left\{\begin{array}{l}
h_{\min }, \quad r<r_{\min }, \\
h_{\min }+\left(h_{\odot}-h_{\min }\right) \frac{\left(r-r_{\min }\right)}{\left(r_{\odot}-r_{\min }\right)}, \quad r \geqslant r_{\min },
\end{array}\right.
$$

with $\quad h_{\min }=165 \mathrm{pc}, r_{\min }=5300 \mathrm{pc}, h_{\odot}=247 \mathrm{pc}, \quad$ and $r_{\odot}=8000 \mathrm{pc}$, the last two quantities being the height of the galactic disk at the position of the Sun and the distance of the Sun from the galactic center, respectively.

Finally, as a model for the galactic halo we take the model of Young [15]:

$$
\nu_{\text {halo }}=\mu_{\text {halo }} \frac{\exp \left[-7.669\left(R / R_{e}\right)^{1 / 4}\right]}{\left(R / R_{e}\right)^{7 / 8}}, \quad R>0.2 R_{e} \text {, }
$$

where $R=\left(r^{2}+z^{2}\right)^{1 / 2}$ denotes the distance from the galactic center and $R_{e}=2700 \mathrm{pc}$.

The final piece of information we need before we can calculate the multipole moments $p_{\ell m}$ for such a distribution is the direction to the galactic center and the orientation of the galaxy. It is standard to express this in terms of the equatorial coordinate system [14] in which the $z$ axis is taken along the celestial north pole, the $x$ axis is taken in the direction of the (1950) vernal equinox. $90^{\circ}-\delta$ and $\alpha$ are taken as the spherical polar coordinates corresponding to these axes. In terms of these, the direction to the galactic center is given by

$$
\alpha=265.6^{\circ}, \quad \delta=-28.9^{\circ},
$$

and the direction of the galactic north pole by

$$
\alpha=192.25^{\circ}, \quad \delta=27.4^{\circ} \text {. }
$$

We take the equatorial coordinate system to define our cosmic frame. We now determine $P(\hat{\Omega})$ by choosing a direction $\hat{\Omega}$ and integrating the intensity along that direction. The number of sources in an element of solid angle $d \Omega$ at distance is $D$ from the Earth is proportional to $\nu D^{2} d D d \Omega$. The intensity of the source drops like $1 / D^{2}$ and hence the integrated intensity is just proportional to $\int \nu d D$. In this way we arrive at $p_{\ell m}$ for each model and hence by Eq. (10.5) the minimum value of $\Omega_{\mathrm{gw}}$ necessary for detection of each multipole. To ensure convergence of the sum in this case it was necessary to include contributions to the sum for $\ell$ up to of order 100 (dependent on $m$ ). Tables II and III show the intensity of the stochastic background distributed in this way required to detect multipole moments from $m=0$ to $m=24$ for the four models with $90 \%$ confidence in one year of observation for initial and advanced LIGO, respectively. 


\section{CONCLUSION}

In this paper, we have shown how the signals from a pair of gravitational wave detectors may be analyzed to search for anisotropies in the stochastic gravitational wave background. We have shown how the correlation between two detectors may be determined with an averaging time short compared to a day but long compared to the light travel time between the detectors, and how this correlation may be decomposed into harmonics of Earth's rotation. We have calculated the signalto-noise ratios associated with such measurements, and shown that certain types of anisotropy might reasonably be detected with instruments that will be available in the nottoo-distant future. For example the anisotropy due to our motion with respect to the cosmological rest frame might well be observable with a second-generation LIGO detector.

At this point, it is difficult to proceed further without a more detailed knowledge of the instrumental data that will be forthcoming. The results given in this paper make it straightforward to predict the expected harmonic amplitudes $S_{m}$ of the detector correlation for a given anisotropic distribution $p_{\ell m}$ of gravitational wave background. It is more difficult to go the other way. This would involve using a given set of observed harmonic amplitudes $S_{m}$ to obtain the values of (or constraints on) the $p_{\ell m}$. There are a variety of fitting techniques that could be used-making the appropriate choice will probably require real data.

\section{ACKNOWLEDGMENTS}

This work has been partially supported by the National Science Foundation Grant No. PHY95-07740, by the Forbairt Grant No. SC/96/712, and by the LIGO visitors Program No. PHY92-10038.

\section{APPENDIX: ANALYTIC EVALUATION OF INTEGRALS}

In this appendix we derive a closed form expression for the integral

$$
\mathcal{I}^{k}(x)=\int_{-1}^{1} d u e^{i u x} P^{k}(u) u^{N}\left(1-u^{2}\right)^{|k| / 2},
$$

where $N$ is a non-negative integer.

First we note that

$$
P_{\ell}^{-k}(u)=(-1)^{k} \frac{(l-k) !}{(l+k) !} P^{k}(u)
$$

so that it is only necessary to deal with the case $k \geqslant 0$. For $k \geqslant 0$ we have

$$
P^{k}(u)=(-1)^{k}\left(1-u^{2}\right)^{k / 2} \frac{d^{k}}{d u^{k}} P_{\ell}(u)
$$

giving

$$
\mathcal{I}^{k}(x)=(-1)^{k} \int_{-1}^{1} d u e^{i u x} u^{N}\left(1-u^{2}\right)^{k} \frac{d^{k}}{d u^{k}} P_{\curlywedge}(u) .
$$

The presence of the factor $\left(1-u^{2}\right)^{k}$ now ensures that when we integrate by parts $k$ times no boundary terms appear so that

$$
\mathcal{I}_{\ell}^{k}(x)=\int_{-1}^{1} d u P(u) \frac{d^{k}}{d u^{k}}\left[e^{i u x} u^{N}\left(1-u^{2}\right)^{k}\right]
$$

The derivative can be expanded by the Liebniz rule to give

$$
\sum_{r=0}^{k}(i x)^{r} p_{N+k+r}(u) e^{i u x}
$$

where $p_{N+k+r}(u)$ is a polynomial of degree $N+k+r$. Our problem is thus reduced to that of finding

$$
\mathcal{J}_{\ell}^{M}(x)=\int_{-1}^{1} d u e^{i u x} u^{M} P_{\ell}(u) .
$$

For $M=0$ this is an elementary integral given by

$$
\mathcal{J}_{\ell}^{0}(x)=2 i^{\ell} j \ell(x) \text {, }
$$

where $j \ell(x)$ denotes the spherical Bessel function of order $\ell$ [16]. Higher $M$ values may then be obtained by differentiation

$$
\mathcal{J}_{\ell}^{M}(x)=(-i)^{M} \frac{d^{M}}{d x^{M}} \mathcal{J}_{\ell}^{0}(x) .
$$

These derivatives may in turn be expressed back in terms of (undifferentiated) spherical Bessel functions using the relations [16]

$$
\begin{aligned}
\frac{d}{d x} j \ell(x) & =\frac{1}{2 \ell+1}\left[\ell_{\ell-1}(x)-(\ell+1) j_{\ell+1}(x)\right] \\
& =\frac{\ell}{x} j(x)-j_{\ell+1}(x) .
\end{aligned}
$$

For $M=1$ to 8 we have

$$
\begin{gathered}
\mathcal{J}_{\ell}^{1}(x)=\frac{\ell}{(2 \ell+1)} j_{\ell-1}(x)-\frac{(\ell+1)}{(2 \ell+1)} j_{\ell+1}(x), \\
\mathcal{J}^{2}(x)=\frac{(\ell-1) \ell}{(2 \ell-1)(2 \ell+1)} j_{\ell-2}(x)-\frac{\left(2 \ell^{2}+2 \ell-1\right)}{(2 \ell-1)(2 \ell+3)} j \ell(x)+\frac{(\ell+1)(\ell+2)}{(2 \ell+1)(2 \ell+3)} j \ell+2(x), \\
\mathcal{J}^{3}(x)=\frac{\ell(\ell-1)(\ell-2)}{(2 \ell-3)(2 \ell-1)(2 \ell+1)} j \ell-3(x)-\frac{3 \ell\left(\ell^{2}-2\right)}{(2 \ell-3)(2 \ell+1)(2 \ell+3)} j \ell-1(x)+\frac{3(\ell+1)\left(\ell^{2}+2 \ell-1\right)}{(2 \ell-1)(2 \ell+1)(2 \ell+5)} j \ell+1(x) \\
-\frac{(\ell+1)(\ell+2)(\ell+3)}{(2 \ell+1)(2 \ell+3)(2 \ell+5)} j_{\ell+3}(x),
\end{gathered}
$$




$$
\begin{aligned}
& \mathcal{J}^{4}(x)=\frac{\ell(\ell-1)(\ell-2)(\ell-3)}{(2 \ell+1)(2 \ell-1)(2 \ell-3)(2 \ell-5)} j_{\ell-4}(x)-\frac{\left.2 \ell(\ell-1)\left(2 \ell^{2}-2 \ell-7\right)\right)}{(2 \ell+3)(2 \ell+1)(2 \ell-1)(2 \ell-5)} j \ell-2(x) \\
& +\frac{3\left(2 \ell^{4}+4 \ell^{3}-6 \ell^{2}-8 \ell+3\right)}{(2 \ell+5)(2 \ell+3)(2 \ell-1)(2 \ell-3)} j \ell(x)-\frac{2(\ell+1)(\ell+2)\left(2 \ell^{2}+6 \ell-3\right)}{(2 \ell+7)(2 \ell+3)(2 \ell+1)(2 \ell-1)} j \ell+2(x) \\
& +\frac{(\ell+1)(\ell+2)(\ell+3)(\ell+4)}{(2 \ell+7)(2 \ell+5)(2 \ell+3)(2 \ell+1)} j_{\ell+4}(x) \\
& \mathcal{J}^{5}(x)=\frac{\ell(\ell-1)(\ell-2)(\ell-3)(\ell-4)}{(2 \ell-7)(2 \ell-5)(2 \ell-3)(2 \ell-1)(2 \ell+1)} j \ell-5(x)-\frac{5 \ell(\ell-1)(\ell-2)\left(\ell^{2}-2 \ell-5\right)}{(2 \ell-7)(2 \ell-3)(2 \ell-1)(2 \ell+1)(2 \ell+3)} j \ell-3(x) \\
& +\frac{5 \ell\left(2 \ell^{4}-16 \ell^{2}+23\right)}{(2 \ell-5)(2 \ell-3)(2 \ell+1)(2 \ell+3)(2 \ell+5)} j \ell-1(x)-\frac{5(\ell+1)\left(2 \ell^{4}+8 \ell^{3}-4 \ell^{2}-24 \ell+9\right)}{(2 \ell-3)(2 \ell-1)(2 \ell+1)(2 \ell+5)(2 \ell+7)} j \ell+1(x) \\
& +\frac{5(\ell+1)(\ell+2)(\ell+3)\left(\ell^{2}+4 \ell-2\right)}{(2 \ell-1)(2 \ell+1)(2 \ell+3)(2 \ell+5)(2 \ell+9)} j \ell+3(x)-\frac{(\ell+1)(\ell+2)(\ell+3)(\ell+4)(\ell+5)}{(2 \ell+1)(2 \ell+3)(2 \ell+5)(2 \ell+7)(2 \ell+9)} j \ell+5(x), \\
& \mathcal{J}^{6}(x)=\frac{\ell(\ell-1)(\ell-2)(\ell-3)(\ell-4)(\ell-5)}{(2 \ell-9)(2 \ell-7)(2 \ell-5)(2 \ell-3)(2 \ell-1)(2 \ell+1)} j \ell-6(x) \\
& -\frac{3 \ell(\ell-1)(\ell-2)(\ell-3)\left(2 \ell^{2}-6 \ell-13\right)}{(2 \ell-9)(2 \ell-5)(2 \ell-3)(2 \ell-1)(2 \ell+1)(2 \ell+3)} j \ell-4(x) \\
& +\frac{15 \ell(\ell-1)\left(\ell^{4}-2 \ell^{3}-11 \ell^{2}+12 \ell+29\right)}{(2 \ell-7)(2 \ell-5)(2 \ell-1)(2 \ell+1)(2 \ell+3)(2 \ell+5)} j \ell-2(x) \\
& -\frac{5\left(4 \ell^{6}+12 \ell^{5}-38 \ell^{4}-96 \ell^{3}+88 \ell^{2}+138 \ell-45\right)}{(2 \ell-5)(2 \ell-3)(2 \ell-1)(2 \ell+3)(2 \ell+5)(2 \ell+7)} j \ell(x) \\
& +\frac{15(\ell+1)(\ell+2)\left(\ell^{4}+6 \ell^{3}+\ell^{2}-24 \ell+9\right)}{(2 \ell-3)(2 \ell-1)(2 \ell+1)(2 \ell+3)(2 \ell+7)(2 \ell+9)} j \ell+2(x) \\
& -\frac{3(\ell+1)(\ell+2)(\ell+3)(\ell+4)\left(2 \ell^{2}+10 \ell-5\right)}{(2 \ell-1)(2 \ell+1)(2 \ell+3)(2 \ell+5)(2 \ell+7)(2 \ell+11)} j \ell+4(x) \\
& +\frac{(\ell+1)(\ell+2)(\ell+3)(\ell+4)(\ell+5)(\ell+6)}{(2 \ell+1)(2 \ell+3)(2 \ell+5)(2 \ell+7)(2 \ell+9)(2 \ell+11)} j \ell+6(x), \\
& \mathcal{J}^{7}(x)=\frac{\ell(\ell-1)(\ell-2)(\ell-3)(\ell-4)(\ell-5)(\ell-6)}{(2 \ell-11)(2 \ell-9)(2 \ell-7)(2 \ell-5)(2 \ell-3)(2 \ell-1)(2 \ell+1)} j \ell-7(x) \\
& -\frac{7 \ell(\ell-1)(\ell-2)(\ell-3)(\ell-4)\left(\ell^{2}-4 \ell-8\right)}{(2 \ell-11)(2 \ell-7)(2 \ell-5)(2 \ell-3)(2 \ell-1)(2 \ell+1)(2 \ell+3)} j \ell-5(x) \\
& +\frac{21 \ell(\ell-1)(\ell-2)\left(\ell^{4}-4 \ell^{3}-12 \ell^{2}+32 \ell+54\right)}{(2 \ell-9)(2 \ell-7)(2 \ell-3)(2 \ell-1)(2 \ell+1)(2 \ell+3)(2 \ell+5)} j \ell-3(x) \\
& -\frac{35 \ell\left(\ell^{2}-6\right)\left(\ell^{4}-14 \ell^{2}+22\right)}{(2 \ell-7)(2 \ell-5)(2 \ell-3)(2 \ell+1)(2 \ell+3)(2 \ell+5)(2 \ell+7)} j \ell-1(x) \\
& +\frac{35(\ell+1)\left(\ell^{2}+2 \ell-5\right) *\left(\ell^{4}+4 \ell^{3}-8 \ell^{2}-24 \ell+9\right)}{(2 \ell-5)(2 \ell-3)(2 \ell-1)(2 \ell+1)(2 \ell+5)(2 \ell+7)(2 \ell+9)} j \ell+1(x) \\
& -\frac{21(\ell+1)(\ell+2)(\ell+3)\left(\ell^{4}+8 \ell^{3}+6 \ell^{2}-40 \ell+15\right)}{(2 \ell-3)(2 \ell-1)(2 \ell+1)(2 \ell+3)(2 \ell+5)(2 \ell+9)(2 \ell+11)} j \ell+3(x) \\
& +\frac{7(\ell+1)(\ell+2)(\ell+3)(\ell+4)(\ell+5)\left(\ell^{2}+6 \ell-3\right)}{(2 \ell-1)(2 \ell+1)(2 \ell+3)(2 \ell+5)(2 \ell+7)(2 \ell+9)(2 \ell+13)} j \ell+5(x) \\
& (\ell+1)(\ell+2)(\ell+3)(\ell+4)(\ell+5)(\ell+6)(\ell+7) \\
& \frac{(2 \ell+1)(2 \ell+3)(2 \ell+5)(2 \ell+7)(2 \ell+9)(2 \ell+11)(2 \ell+13)}{(\ell+7} j(x) \text {, }
\end{aligned}
$$




$$
\begin{aligned}
& \mathcal{J}_{\ell}(x)=\frac{\ell(\ell-1)(\ell-2)(\ell-3)(\ell-4)(\ell-5)(\ell-6)(\ell-7)}{(2 \ell-13)(2 \ell-11)(2 \ell-9)(2 \ell-7)(2 \ell-5)(2 \ell-3)(2 \ell-1)(2 \ell+1)} j_{\ell-8}(x) \\
& -\frac{4 \ell(\ell-1)(\ell-2)(\ell-3)(\ell-4)(\ell-5)\left(2 \ell^{2}-10 \ell-19\right)}{(2 \ell-13)(2 \ell-9)(2 \ell-7)(2 \ell-5)(2 \ell-3)(2 \ell-1)(2 \ell+1)(2 \ell+3)} j \ell-6(x) \\
& +\frac{14 \ell(\ell-1)(\ell-2)(\ell-3)\left(2 \ell^{4}-12 \ell^{3}-22 \ell^{2}+120 \ell+173\right)}{(2 \ell-11)(2 \ell-9)(2 \ell-5)(2 \ell-3)(2 \ell-1)(2 \ell+1)(2 \ell+3)(2 \ell+5)} j \ell-4(x) \\
& -\frac{28 \ell(\ell-1)\left(2 \ell^{6}-6 \ell^{5}-49 \ell^{4}+108 \ell^{3}+371 \ell^{2}-426 \ell-873\right)}{(2 \ell-9)(2 \ell-7)(2 \ell-5)(2 \ell-1)(2 \ell+1)(2 \ell+3)(2 \ell+5)(2 \ell+7)} j \ell-2(x) \\
& +\frac{35\left(2 \ell^{8}+8 \ell^{7}-44 \ell^{6}-160 \ell^{5}+286 \ell^{4}+848 \ell^{3}-604 \ell^{2}-1056 \ell+315\right)}{(2 \ell-7)(2 \ell-5)(2 \ell-3)(2 \ell-1)(2 \ell+3)(2 \ell+5)(2 \ell+7)(2 \ell+9)} j \ell(x) \\
& -\frac{28(\ell+1)(\ell+2)\left(2 \ell^{6}+18 \ell^{5}+11 \ell^{4}-204 \ell^{3}-157 \ell^{2}+690 \ell-225\right)}{(2 \ell-5)(2 \ell-3)(2 \ell-1)(2 \ell+1)(2 \ell+3)(2 \ell+7)(2 \ell+9)(2 \ell+11)} j \ell+2(x) \\
& +\frac{14(\ell+1)(\ell+2)(\ell+3)(\ell+4)\left(2 \ell^{4}+20 \ell^{3}+26 \ell^{2}-120 \ell+45\right)}{(2 \ell-3)(2 \ell-1)(2 \ell+1)(2 \ell+3)(2 \ell+5)(2 \ell+7)(2 \ell+11)(2 \ell+13)} j \ell+4(x) \\
& -\frac{4(\ell+1)(\ell+2)(\ell+3)(\ell+4)(\ell+5)(\ell+6)\left(2 \ell^{2}+14 \ell-7\right)}{(2 \ell-1)(2 \ell+1)(2 \ell+3)(2 \ell+5)(2 \ell+7)(2 \ell+9)(2 \ell+11)(2 \ell+15)} j \ell+6(x) \\
& +\frac{(\ell+1)(\ell+2)(\ell+3)(\ell+4)(\ell+5)(\ell+6)(\ell+7)(\ell+8)}{(2 \ell+1)(2 \ell+3)(2 \ell+5)(2 \ell+7)(2 \ell+9)(2 \ell+11)(2 \ell+13)(2 \ell+15)} j \ell+8(x) .
\end{aligned}
$$

Alternative forms may by obtained by use of Eq. (A10).

[1] A. Abramovici et al., Science 256, 325 (1992).

[2] B. Caron et al., in Gravitational Wave Experiments, Proceedings of the Edoardo Amaldi Conference (World Scientific, Singapore, 1995), p. 86.

[3] K. Danzmann et al., in Gravitational Wave Experiments, Proceedings of the Edoardo Amaldi Conference (World Scientific, Singapore, 1995), p. 100.

[4] K. Tsubono et al., in Gravitational Wave Experiments, Proceedings of the Edoardo Amaldi Conference (World Scientific, Singapore, 1995), p. 112.

[5] P. F. Michelson, Mon. Not. R. Astron. Soc. 227, 933 (1987).

[6] N. Christensen, Phys. Rev. D 46, 5250 (1992).

[7] E. Flanagan, Phys. Rev. D 48, 2389 (1993). Note that the second term on the right-hand side of Eq. (B6) should read $-10 j_{1}(\alpha)$ rather than $-2 j_{1}(\alpha)$, and that the sliding delay function shown in Fig. 2 on p. 2394 is incorrect.

[8] B. Allen, in Proceedings of the Les Houches School on Astrophysical Sources of Gravitational Radiation, edited by J. A. Marck and J. P. Lasota (Cambridge University Press, Cam- bridge, England, in press).

[9] G. Arfken, Mathematical Methods for Physicists, 3rd ed. (Academic, Orlando, 1985). Note that this reference uses different conventions for the normalization of associated Legendre functions $\mathrm{P}_{l}^{m}$ than the other references here, however, the spherical harmonic functions $Y_{\ell m}$ are identical to the other references.

[10] M. E. Rose, Elementary Theory of Angular Momentum (Wiley, New York, 1957).

[11] I. S. Gradshteyn and I. M. Ryzhik, Table of Integrals, Series and Products, 4th ed. (Academic, San Diego, 1980).

[12] Particle Data Group, R. M. Barnett et al., Phys. Rev. D 54, 1 (1996), p. 118.

[13] S. M. Kent, T. M. Dame, and G. Fazio, Astrophys. J. 378, 131 (1991).

[14] D. Mihalas and J. Binney, Galactic Astronomy, 2nd ed. (Freeman, San Francisco, 1981)

[15] P. J. Young, Astron. J. 81, 807 (1976).

[16] J. D. Jackson, Classical Electrodynamics, 2nd ed. (Wiley, New York, 1975). 